TRANSACTIONS OF THE

AMERICAN MATHEMATICAL SOCIETY

Volume 360, Number 1, January 2008, Pages 307-329

S 0002-9947(07)04263-8

Article electronically published on July 23, 2007

\title{
STEIN COMPACTS IN LEVI-FLAT HYPERSURFACES
}

\author{
FRANC FORSTNERIČ AND CHRISTINE LAURENT-THIÉBAUT
}

\begin{abstract}
We explore connections between geometric properties of the Levi foliation of a Levi-flat hypersurface $M$ and holomorphic convexity of compact sets in $M$, or bounded in part by $M$. Applications include extendability of Cauchy-Riemann functions, solvability of the $\bar{\partial}_{b}$-equation, approximation of Cauchy-Riemann and holomorphic functions, and global regularity of the $\bar{\partial}$ Neumann operator.
\end{abstract}

\section{INTRODUCTION}

A real hypersurface $M$ in an $n$-dimensional complex manifold is said to be Leviflat if it is foliated by complex manifolds of dimension $n-1$; this Levi foliation is as smooth as $M$ according to Barrett and Fornæss [9]. A Levi-flat hypersurface locally partitions the complex manifold into two subsets, both of which are pseudoconvex along the hypersurface. Such hypersurfaces have recently received a lot of attention; see, e.g., [29], 49], [51, [58].

In this paper we describe a connection between the geometric properties of the Levi foliation and the complex analytic properties of certain compact sets in a Levi-flat hypersurface. We indicate applications ranging from the approximation of Cauchy-Riemann and holomorphic functions, the exact solvability of the $\bar{\partial}_{b^{-}}$ equation (without shrinking the domain), to the global regularity of the $\bar{\partial}$-Neumann operator on certain pseudoconvex domains in $\mathbb{C}^{n}$ containing a Levi-flat patch in the boundary.

In many analytic problems it is important to know that a certain compact set in a complex manifold admits a basis of open Stein neighborhoods; such a set will be called a Stein compact (34], 38]). Sometimes one needs Stein neighborhoods with certain additional analytic or topological properties. A theorem of Siu [57] implies that each topologically closed leaf in a Levi-flat hypersurface which is exhausted by a strongly plurisubharmonic function admits a basis of open Stein neighborhoods in the ambient complex manifold (see also Colţoiu [20] and Demailly [22]). Globally the situation is more complicated and far from well understood.

One possible approach is to look for a holomorphic vector field transverse to $M$ on a given compact; its flow translates $M$ to a family of Levi-flat hypersurfaces, thus providing Stein neighborhoods of the original set. This approach was used by Bedford and Fornæss 11 to find precise results for complex curves in pseudoconvex

Received by the editors March 17, 2005 and, in revised form, February 7, 2006.

2000 Mathematics Subject Classification. Primary 32D15, 32T20, 32T27, 32V05, 32V25; Secondary 57R30.

Key words and phrases. Levi-flat hypersurfaces, foliations, Stein manifolds.

The first author was supported by grants P1-0291 and J1-6173, Republic of Slovenia.

(C) 2007 American Mathematical Society

Reverts to public domain 28 years from publication 
boundaries, but so far it has been less successful in the case of Levi-flat hypersurfaces. Here we use a different method. Our first main result is the following.

Theorem 1.1. Assume that $M$ is an orientable Levi-flat hypersurface of class $\mathcal{C}^{3}$ in a complex manifold $X$. Let $\rho$ be a strongly plurisubharmonic $\mathcal{C}^{2}$ function in an open set $U \subset X$ such that the set $A=\{x \in U \cap M: \rho(x) \leq 0\}$ is compact. (Such an $A$ will be called a compact strongly pseudoconvex set in $M$.) If the Levi foliation of $M$ is defined in a neighborhood of $A$ by a nowhere vanishing closed one-form of class $\mathcal{C}^{2}$, then $A$ is a Stein compact.

If we assume in addition that $d\left(\left.\rho\right|_{M}\right) \neq 0$ on $\{x \in M \cap U: \rho(x)=0\}=b A$, then $A$ is even uniformly $\mathcal{H}$-convex (Theorem 1.4 and Proposition 3.3).

Theorem 1.1 is proved in $\S 3$. First we show that the existence of a closed oneform defining the Levi foliation of $M$ is equivalent to the existence of a $\mathcal{C}^{3}$ defining function $v$ for $M$ such that $d d^{c} v=2 i \partial \bar{\partial} v$ and its first order derivatives vanish at every point of $M$ (Proposition 3.1). Such an asymptotically pluriharmonic defining function is used to find a Stein neighborhood basis of $A$ (Propositions 3.3 and 3.5). With more regularity of $M$ we also obtain transverse holomorphic vector fields (Proposition 17.1). The restriction to compacts is essential; for example, the Leviflat hypersurface $M=\mathbb{C} \times \mathbb{R} \subset \mathbb{C}^{2}$ does not admit a basis of Stein neighborhoods in $\mathbb{C}^{2}[3]$.

For the record we state here several sufficient conditions for the existence of a nonvanishing closed one-form defining a codimension one foliation; they are obtained from the classical theory of Reeb, Haefliger, Sullivan, Thurston and others $(\S 4-\S 6)$.

Proposition 1.2. A transversely orientable codimension one foliation $\mathcal{L}$ of class $\mathcal{C}^{r}(r \geq 2)$ on a $\mathcal{C}^{r}$ manifold $M$ is defined in a neighborhood of a certain compact set $A \subset M$ by a closed nowhere vanishing one-form of class $\mathcal{C}^{r-1}$ provided that any one of the following conditions holds:

(a) $\mathcal{L}$ is a simple foliation in a neighborhood of $A$ (every point admits a local transversal to $\mathcal{L}$ intersecting each leaf at most once).

(b) There is an open neighborhood $U \subset M$ of $A$ such that every leaf of the restricted foliation $\left.\mathcal{L}\right|_{U}$ is topologically closed in $U$.

(c) $\mathcal{L}$ has no nontrivial one-sided holonomy (this is the case when $M$ is real analytic, or when the leaves are simply connected), and each element of $\pi_{1}(M)$ is of finite order.

(d) $M$ is compact and there is a compact leaf $L \in \mathcal{L}$ with $H^{1}(L, \mathbb{R})=0$.

(e) $H^{1}\left(A, \mathcal{C}_{\mathcal{L}}^{r-1}\right)=0$ where $\mathcal{C}_{\mathcal{L}}^{r-1}$ is the sheaf of real valued $\mathcal{C}^{r-1}$ functions on $M$ which are constant on the leaves of $\mathcal{L}$.

The sufficient conditions in Theorem 1.1 and Proposition 1.2 seem fairly close to optimal. In $\S 8$ we show, using an example due to Bedford and Fornæss [11, p. 21], that the conclusion of Theorem 1.1 (and of most analytic applications mentioned below) fails in general in the presence of a leaf with nontrivial infinitesimal holonomy. In that example $M$ is a real analytic Levi-flat hypersurface in $\mathbb{C}^{*} \times \mathbb{C}$ whose intersection with a certain compact strongly pseudoconvex domain fails to be a Stein compact, and both sides of $M$ are worm domains in the sense of Diederich and Fornæss 23.

In 63. Straube and Sucheston constructed closed one-forms defining certain Levi foliations on a compact hypersurface with boundary $M \subset \mathbb{C}^{2}$, assuming that the 
leaves are closed and the foliation satisfies certain geometric conditions at $b M$. (See also [62, p. 256, Proposition].) Using ideas of Sullivan 64 they characterized the existence of a smooth closed one-form defining the foliation in terms of foliation currents associated to a transverse flow [63, p. 152, Proposition 2 and Theorem 3].

Condition (c) in Proposition 1.2 implies the following.

Corollary 1.3. If $M$ is a simply connected, real analytic, Levi-flat hypersurface in a complex manifold $X$, then every compact strongly pseudoconvex subset $A \subset M$ is a Stein compact in $X$.

We now indicate some analytic applications of Theorem 1.1 Our original motivation was the problems on extendibility of Cauchy-Riemann (CR) functions and solvability of the $\bar{\partial}_{b}$-equation. Consider the following setup:

(1) $X$ is a Stein manifold of dimension $n \geq 2$,

(2) $D \Subset X$ is a smoothly bounded strongly pseudoconvex domain,

(3) $M$ is a Levi-flat hypersurface in $X$ intersecting $b D$ transversely,

(4) $A=M \cap \bar{D}$, and

(5) $\Omega$ is a connected component of $D \backslash M$ such that $b \Omega=A \cup \omega$, where $\omega$ is an open connected subset of $b D$.

Let $d$ be a distance function on $X$ induced by a smooth Riemannian metric. A compact set $A$ in $X$ is uniformly $\mathcal{H}$-convex if there are open Stein domains $U_{\nu} \subset X$ $(\nu=1,2, \ldots)$ and a number $c \geq 1$ such that

$$
\left\{x \in X: d(x, A)<\frac{1}{c \nu}\right\} \subset U_{\nu} \subset\left\{x \in X: d(x, A)<\frac{c}{\nu}\right\}, \quad \nu \in \mathbb{N} .
$$

The following result follows from Theorem 1.1 and Proposition 3.3

Theorem 1.4. Let $X, M, D, A$ be as in (1)-(4), with bD of class $\mathcal{C}^{2}$. If one of the conditions in Theorem 1.1 or Proposition 1.2 holds, then the set $A$, and also the closure of every connected component of $D \backslash M$, is uniformly $\mathcal{H}$-convex (and hence a Stein compact).

Assuming the above setup (1)-(5) we consider the following analytic conditions; (A2) and (A3) are relevant only if $n \geq 3$.

(A1): Every continuous CR function on $\omega$ extends to a (unique) continuous function on $E(\omega)=\bar{\Omega} \backslash A$ which is holomorphic in $\Omega$.

(A2): For every smooth $(0, q)$-form $f$ on $\omega(1 \leq q \leq n-3)$ satisfying $\bar{\partial}_{b} f=0$ there is a smooth $(0, q-1)$-form $u$ on $\omega$ such that $\bar{\partial}_{b} u=f$.

(A3): For every smooth $(0, n-2)$-form $f$ on $\omega$ for which $\int_{\omega} f \wedge \varphi=0$ for every smooth, $\bar{\partial}$-closed $(n, 1)$-form $\varphi$ on $X \backslash A \operatorname{such}$ that $\operatorname{supp} \varphi \cap \operatorname{supp} f$ is compact, there is a smooth $(0, n-3)$-form $u$ on $\omega$ satisfying $\bar{\partial}_{b} u=f$.

We recall the following result of the second author.

Theorem $1.5([4])$. If $\bar{\Omega}$ is a Stein compact, then

$$
(A 1),(A 2) \text { and }(A 3) \text { hold } \Longleftrightarrow H^{n, q}(A)=0 \text { for all } 1 \leq q \leq n-1 .
$$

Since all Dolbeault cohomology groups of a Stein compact vanish, Theorems 1.1, 1.4 and 1.5 together imply

Corollary 1.6. Assuming the setup (1)-(5), each of the conditions in Theorem 1.1 and Proposition 1.2 implies that (A1), (A2), (A3) hold. 
Results on extendibility of CR functions from caps $\omega \subset b D$ have been obtained in [41, 42, 45], 46], 47]. For solutions of the $\bar{\partial}_{b}$-equation on $\omega$ without estimates up to the boundary, see [44]; for $L^{p}$ and Sobolev estimates, see M.-C. Shaw [54, 55 .

Our next application depends on the results of Boas and Straube [12, [13] on regularity of the $\bar{\partial}$-Neumann operator $N_{q}$ on smoothly bounded pseudoconvex domains $\Omega \Subset \mathbb{C}^{n}$. (See also Straube 60, and Straube and Sucheston 62, 63. We thank E. Straube for pointing out this connection.) Recall that $N_{q}$ is the inverse of the complex Laplacian $\square=\bar{\partial}_{\bar{\partial}}^{*}+\bar{\partial}^{*} \bar{\partial}$ acting on $(0, q)$-forms, and the Bergman projection $P_{q}=I-\bar{\partial}^{*} N_{q+1} \bar{\partial}$ is the orthogonal projection from the space of $(0, q)$ forms with $L^{2}$-integrable coefficients onto the subspace of $\bar{\partial}$-closed forms. (For the $\bar{\partial}$-Neumann theory, see [17], [24], 40].) Let $W_{(0, q)}^{s}(\Omega)$ denote the Sobolev space of all $(0, q)$-forms whose coefficients have partial derivatives of order $\leq s$ in $L^{2}(\Omega)$.

Theorem 1.7. Let $\Omega \Subset \mathbb{C}^{n}$ be a smoothly bounded pseudoconvex domain. Assume that the (compact) set $A \subset b \Omega$, consisting of all points of infinite D'Angelo type 21], satisfies the following properties:

(i) $A$ is the closure of its interior in $b \Omega$, and

(ii) $A$ is a strongly pseudoconvex set in a smooth Levi-flat hypersurface $M \subset \mathbb{C}^{n}$. (See Theorem 1.1.)

If the Levi foliation of $M$ is defined in a neighborhood of $A$ by a smooth closed 1-form (in particular, if one of the conditions in Proposition 1.2 holds), then the $\bar{\partial}$ Neumann operator $N_{q}$ and the Bergman projection $P_{q}$ are continuous on $W_{(0, q)}^{s}(\Omega)$ when $0 \leq q \leq n$ and $s \geq 0$.

Theorem 1.7 is proved in $\S 7$. The conclusion may fail if $A$ is not the closure of its relative interior in $b \Omega$; a well-known example is the complex annulus in the boundary of the Diederich-Fornæss worm domain [23] (Barrett [8, Christ [19]). See also the example in $\S 8$ below.

We conclude this introduction with two theorems on holomorphic approximation, which are proved in $\S 7$. The first of them was proposed by G. Tomassini (private communication). Note that a function on a Levi-flat hypersurface is a CR function if and only if its restriction to every leaf is holomorphic. Any CR function on a CR submanifold admits local holomorphic approximations [5].

Theorem 1.8. Let $A \subset M \subset X=\mathbb{C}^{n}$ be as in Theorem 1.1. Assume that $M$ is of class $\mathcal{C}^{r}, r \geq n+2$, and its Levi foliation is defined in a neighborhood of $A$ by a closed one-form of class $\mathcal{C}^{r-1}$. Let $k \in\{0,1, \ldots, r-1\}$ and $0<\alpha<1$. Every $C R$ function of class $\mathcal{C}^{k, \alpha}$ in a neighborhood of $A$ in $M$ can be approximated in the $\mathcal{C}^{k, \alpha}$ sense by functions holomorphic in a neighborhood of $A$ in $X$.

We believe that a version of Theorem 1.8 can also be proved for Levi-flat hypersurfaces in an arbitrary complex manifold since $A$ admits Stein neighborhoods; however, it appears that the smoothness hypothesis on $M$ should be higher, and for simplicity we restrict ourselves to $X=\mathbb{C}^{n}$.

The final result depends on a theorem of Fornæss and Nagel [25]; we thank E. Straube for calling this to our attention. 
Theorem 1.9. Let $\Omega \Subset \mathbb{C}^{n}$ be a smoothly bounded pseudoconvex domain and $A$ a compact subset of $b \Omega$ satisfying the following:

(i) $A$ is the closure of its interior in $b \Omega$,

(ii) $b \Omega \backslash A$ is strongly pseudoconvex, and

(iii) $A$ is a compact strongly pseudoconvex set in a smooth Levi-flat hypersurface $M \subset \mathbb{C}^{n}$ (see Theorem 1.1).

If the Levi foliation of $M$ is defined in a neighborhood of $A$ by a smooth closed 1-form (in particular, if one of the conditions in Proposition 1.2 holds), then

(1) $\bar{\Omega}$ admits a basis of open Stein neighborhoods in $\mathbb{C}^{n}$, and

(2) every function which is holomorphic in $\Omega$ and continuous on $\bar{\Omega}$ can be approximated uniformly on $\bar{\Omega}$ by functions holomorphic in a neighborhood of $\bar{\Omega}$.

\section{Preliminaries}

We denote by $j^{r} \eta$ the $r$-jet extension of a function or a differential form $\eta$ on a manifold $X$. (In local coordinates on $X, j^{r} \eta$ is the collection of all partial derivatives of order $\leq r$ of the components of $\eta$.) The notation $\left.j^{r} \eta\right|_{M}=0$ will mean that the $r$-jet $j^{r} \eta$ (with respect to all variables in the ambient manifold $X$ ) vanishes at all points $p \in M$.

We recall some relevant notions concerning codimension one foliations and Leviflat hypersurfaces. Our general references for the theory of foliations will be [15, [16, 31, and 65].

Let $M$ be a real manifold of dimension $m$ and class $\mathcal{C}^{r}, r \in\{1, \ldots,+\infty, \omega\}$, where $\mathcal{C}^{\omega}$ means real analytic. A foliation $\mathcal{L}$ of codimension one and class $\mathcal{C}^{r}$ on $M$ is given by a foliation atlas $\mathcal{U}=\left\{\left(U_{j}, \phi_{j}\right): j \in J\right\}$ where $\left\{U_{j}\right\}_{j \in J}$ is a covering of $M$ by open connected sets, for every index $j \in J$ the map $\phi_{j}=\left(\phi_{j}^{\prime}, h_{j}\right): U_{j} \rightarrow$ $P_{j}=P_{j}^{\prime} \times I_{j} \subset \mathbb{R}^{m-1} \times \mathbb{R}$ is a $\mathcal{C}^{r}$ diffeomorphism, and the transition maps are of the form

$$
\theta_{i j}(u, v)=\phi_{i} \circ \phi_{j}^{-1}(u, v)=\left(a_{i j}(u, v), b_{i j}(v)\right)
$$

where $b_{i j}$ is a diffeomorphism between a pair of intervals in $\mathbb{R}$. Each leaf $L \in \mathcal{L}$ intersects $U_{j}$ in at most countably many plaques $\left\{x \in U_{j}: h_{j}(x)=c \in I_{j}\right\}$. The collection $\left\{b_{i j}\right\}$ is called a holonomy cocycle, or a Haefliger cocycle, determining $\mathcal{L}$ [16], 35. A foliation is transversely orientable (resp. transversely real analytic) if it admits a foliation atlas in which all diffeomorphisms $b_{i j}$ preserve the orientation of $\mathbb{R}$ (resp. are real analytic). A continuous function $u: M \rightarrow \mathbb{R}$ is a first integral for $\mathcal{L}$ if $u$ has no local extrema and is constant on every leaf of $\mathcal{L} ; u \in \mathcal{C}^{1}(M)$ is a noncritical first integral if in addition $d u \neq 0$ on $M$.

A closed loop $\gamma$ in a leaf $L \in \mathcal{L}$ determines a germ of a diffeomorphism $\psi_{\gamma}$ (the holonomy of $\gamma$ ) on any local transversal $\ell \subset M$ at a point $x_{0} \in \gamma$, depending only on the homotopy class $[\gamma] \in \pi_{1}\left(L, x_{0}\right)$. The induced map of $\pi_{1}\left(L, x_{0}\right)$ to the group of germs of diffeomorphisms of $\left(\ell, x_{0}\right)$ is called the (germinal) holonomy homomorphism of $L$. $\mathcal{L}$ has trivial leaf holonomy if $\psi_{\gamma}$ is the germ of the identity map for any loop $\gamma$ in a leaf of $\mathcal{L}$; this holds in particular if all leaves are simply connected.

We say that $\mathcal{L}$ admits (nontrivial) one-sided holonomy if the holonomy map $\psi_{\gamma}$ of some leaf $L$, defined on a local transversal $\ell$ at some $p \in L$, equals the identity 
map on one side of $p$ in $\ell$, but not on the other side. A transversely real analytic foliation has no nontrivial one-sided holonomy.

Let $X$ be a complex manifold with the complex structure operator $J$. The operator $d^{c}=-J^{*} d$ is defined on functions by $\left\langle d^{c} v, \xi\right\rangle=-\langle d v, J \xi\rangle$ for $\xi \in T X$. In local holomorphic coordinates $\left(z_{1}, \ldots, z_{n}\right)$, with $z_{j}=x_{j}+i y_{j}$ and $J\left(\frac{\partial}{\partial x_{j}}\right)=\frac{\partial}{\partial y_{j}}$, we have $d^{c} v=\sum_{j=1}^{n}-\frac{\partial v}{\partial y_{j}} d x_{j}+\frac{\partial v}{\partial x_{j}} d y_{j}$. Then $d=\partial+\bar{\partial}, d^{c}=i(\bar{\partial}-\partial)$, and $d d^{c}=2 i \partial \bar{\partial}=\sum_{j, k=1}^{n} \frac{\partial^{2}}{\partial z_{j} \partial \bar{z}_{k}} d z_{j} \wedge d \bar{z}_{k}$ is the Levi-form operator. A function $v$ on $X$ is pluriharmonic if and only if $d d^{c} v=0$.

Let $M=\{v=0\} \subset X$ be a real hypersurface defined by a real function $v \in$ $\mathcal{C}^{r}(X)(r \geq 2)$ with $d v \neq 0$ on $M$. The set $T^{\mathbb{C}} M=T M \cap J(T M)=T M \cap \operatorname{ker} d^{c} v$ is a real codimension one subbundle of $T M$. The Levi form of $M$ is the quadratic $\operatorname{map} T^{\mathbb{C}} M \rightarrow \mathbb{R}$ given by

$$
T^{\mathbb{C}} M \ni \xi \rightarrow\left\langle d d^{c} v, \xi \wedge J \xi\right\rangle=-\left\langle d^{c} v,[\xi, J \xi]\right\rangle .
$$

(The last expression, which follows by Cartan's formula, assumes that $\xi$ is a vector field tangent to $T^{\mathbb{C}} M$.) The hypersurface $M$ is Levi-flat if this form vanishes identically; this is equivalent to $d d^{c} v \wedge d^{c} v=0$ on $T M$, which is the integrability condition for $T^{\mathbb{C}} M=\operatorname{ker}\left(\left.d^{c} v\right|_{T M}\right)$. By Frobenius' integrability theorem the latter is equivalent to the existence of a codimension one foliation of $M$ by submanifolds tangent to $T^{\mathbb{C}} M$, that is, by complex hypersurfaces. For Levi-flat hypersurfaces of low regularity, see [2] and [56].

\section{Asymptotically PluRiharmonic Functions And Stein COMpaCts}

In this section we prove Theorem 1.1. We begin by preparatory results.

Proposition 3.1. Let $M$ be an oriented, closed, Levi-flat hypersurface of class $\mathcal{C}^{r}$ $(r \geq 2)$ in a complex manifold $X$. The following are equivalent:

(i) $M$ admits a defining function $v \in \mathcal{C}^{r}(X)$ with $\left.j^{r-2}\left(d d^{c} v\right)\right|_{M}=0$.

(ii) The Levi foliation of $M$ is defined by a closed one-form of class $\mathcal{C}^{r-1}$.

If $M$ is simply connected, then (i) and (ii) are further equivalent to

(iii) There exists $f=u+i v \in \mathcal{C}^{r}(X)$ such that $M=\{v=0\}, d v \neq 0$ on $M$, and $\left.j^{r-1}(\bar{\partial} f)\right|_{M}=0$.

The equivalences in Proposition 3.1 also hold in the real analytic category (see Remark 3.2). In that case (i) means that $M$ is defined by a function $v$ which is pluriharmonic in a neighborhood of $M$ in $X$, and the function $f=u+i v$ in (iii) is holomorphic in a neighborhood of $M$.

A function $f$ satisfying $\left.j^{r-1}(\bar{\partial} f)\right|_{M}=0$ is said to be asymptotically holomorphic of order $r-1$ on $M$, and a function $v$ satisfying $\left.j^{r-2}\left(d d^{c} v\right)\right|_{M}=0$ is asymptotically pluriharmonic of order $r-2$ on $M$. Since integrability of the subbundle $T^{\mathbb{C}} M$ is equivalent to $\left.d^{c} v \wedge d d^{c} v\right|_{T M}=0$ for some (and hence any) defining function $v$ for $M$ $(\S 2)$, a hypersurface $M=\{v=0\}$ with a defining function satisfying $\left.d d^{c} v\right|_{T M}=0$ is necessarily Levi-flat.

Proof. (i) $\Rightarrow($ ii): Denote by $\iota: M \hookrightarrow X$ the inclusion map. Let $\mathcal{L}$ denote the Levi foliation of $M$, with the tangent bundle $T \mathcal{L}=T^{\mathbb{C}} M$. Assume that $v \in \mathcal{C}^{r}(X)$ satisfies $\left.d v\right|_{M} \neq 0$ and $\left.d d^{c} v\right|_{M}=0$. From $0=\iota^{*}\left(d d^{c} v\right)=d_{M}\left(\iota^{*} d^{c} v\right)$ we see that the one-form $\eta=\iota^{*}\left(d^{c} v\right)$ is closed on $M$. Also, ker $\eta=T^{\mathbb{C}} M=T \mathcal{L}$ and hence $\eta$ defines 
the Levi foliation. Indeed, for $\xi \in T_{x} M$ we have $\langle\eta, \xi\rangle=\left\langle d^{c} v, \xi\right\rangle=-\langle d v, J \xi\rangle$, which is zero if and only if $J \xi \in \operatorname{ker} d v_{x}=T_{x} M$, i.e., when $\xi \in T_{x}^{\mathbb{C}} M$.

(ii) $\Rightarrow$ (i): Let $T \mathcal{L}=\operatorname{ker} \eta$ where $\eta$ is a closed one-form of class $\mathcal{C}^{r-1}$ on $M$. Locally near a point $p \in M$ we have $\eta=-d u$ for some $\mathcal{C}^{r}$ function $u$ which is unique up to an additive real constant. Denoting by $\iota_{L}: L \hookrightarrow M$ the inclusion of a leaf, we have $d\left(\left.u\right|_{L}\right)=\iota_{L}^{*}(d u)=\iota_{L}^{*} \eta=0$. This shows that $u$ is constant on each leaf of $\mathcal{L}$ and hence a Cauchy-Riemann (CR) function on $M$. Hence $u$ admits a $\mathcal{C}^{r}$ extension $f=u+i v$ to a neighborhood $U \subset X$ of $p$ such that $\left.j^{r-1} \bar{\partial} f\right|_{M \cap U}=0$; such an $f$ is unique up to a term which is flat to order $r$ along $M$ ([14, p. 147], Theorem 2 and the remark following it). Since $d d^{c}=2 i \partial \bar{\partial}$, the above implies $\left.j^{r-2} d d^{c}(u+i v)\right|_{M \cap U}=0$. As $d d^{c}$ is a real operator, it follows that $\left.j^{r-2} d d^{c} u\right|_{M \cap U}=0$ and $\left.j^{r-2} d d^{c} v\right|_{M \cap U}=0$. Thus $v$ satisfies condition (i) on $M \cap U$. Since any two local solutions $v$ obtained in this way differ only by a term which is $r$-flat on $M$, we obtain a global solution $v$ by a smooth partition of unity. In the real analytic case the local pluriharmonic solutions are unique and no patching is needed.

If $M$ is simply connected and $T \mathcal{L}=\operatorname{ker} \eta$ for some one-form $\eta$ of class $\mathcal{C}^{r-1}$ with $d \eta=0$, we have $\eta=-d u$ for some $u \in \mathcal{C}^{r}(M)$. The same argument as above gives an asymptotically holomorphic extension $f=u+i v$ into a neighborhood of $M$ in $X$ (holomorphic in the real analytic case), thus showing the equivalence of (iii) with (i) and (ii).

Remark 3.2. The equivalence (i) $\Leftrightarrow($ ii) in Proposition 3.1 was proved in the real analytic case by D. Barrett [7, p. 461, Proposition 1]. For $r=2$ it also follows from results of Straube and Sucheston (combine the equivalence (i) $\Leftrightarrow$ (iv) in 62, p. 252, Theorem] (in the exact case with $\epsilon=0$ ) and [63, p. 152, Proposition 2]).

The equivalent conditions in Proposition 3.1 hold in a small neighborhood of any point in $M$ : take a local $\mathcal{C}^{r}$ first integral $u$ of $\mathcal{L}$ and extend it to a $\bar{\partial}$-flat function $f=u+i v$ as above. Such an $f$ gives a local asymptotically holomorphic flattening of $M$ which in general cannot be chosen to be holomorphic on any side of $M$ by [10]. A real analytic Levi-flat hypersurface need not admit a holomorphic flattening on large domains, not even on contractible ones [27].

Theorem 1.1 will follow from Proposition 3.1 and the following result.

Proposition 3.3. Let $M$ be a Levi-flat hypersurface of class $\mathcal{C}^{3}$ in a complex manifold $X$ and $\rho$ a function of class $\mathcal{C}^{2}$ in an open set $U \subset X$ such that

(i) the set $A=\{x \in U \cap M: \rho(x) \leq 0\}$ is compact,

(ii) $d\left(\left.\rho\right|_{M}\right) \neq 0$ at every point of $b A=\{x \in M \cap U: \rho(x)=0\}$, and

(iii) $d d^{c} \rho>0$ on $T_{x}^{\mathbb{C}} M$ at each point $x \in A$.

If $M \cap U$ admits a defining function $v \in \mathcal{C}^{3}(U)$ satisfying $\left.j^{1}\left(d d^{c} v\right)\right|_{A}=0$, then $A$ is uniformly $\mathcal{H}$-convex and hence a Stein compact.

Proof. Choose a smooth Riemannian metric on $X$ and denote by $|\xi|$ the associated norm of any tangent vector $\xi \in T X$. Since $d d^{c} \rho>0$ on $T^{\mathbb{C}} M$ at the points of $A$, the function $\widetilde{\rho}=\rho+C v^{2}$ is strongly plurisubharmonic in an open neighborhood of $A$ in $X$ provided that the constant $C>0$ is chosen sufficiently large. Replacing $\rho$ by $\widetilde{\rho}$ and shrinking $U$ around $A$ we may therefore assume that the quantities $|d v|$ and $d d^{c} \rho>0$ are bounded, and also bounded away from zero, on $U$. The distance of a point $x \in U$ to $M$ is comparable to $|v(x)|$. 


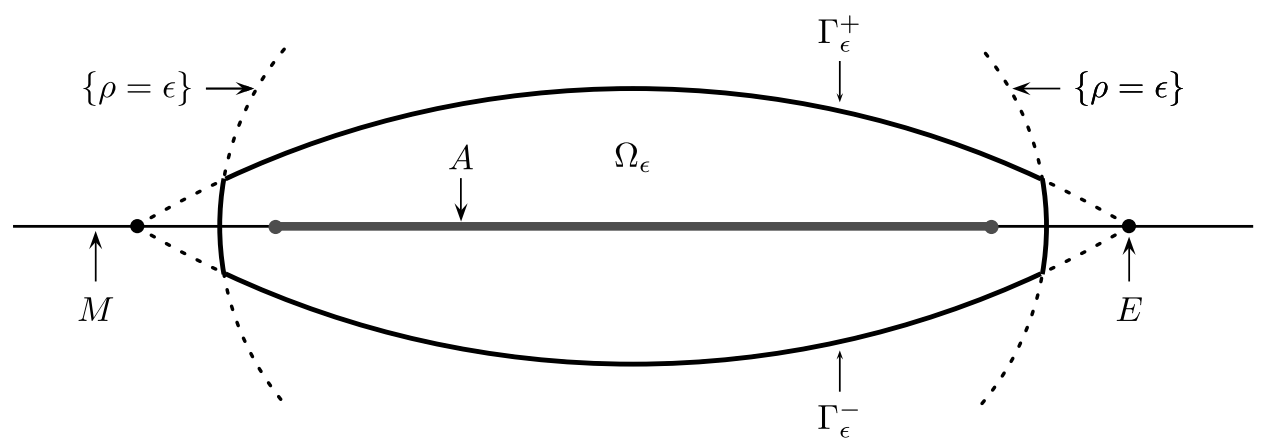

FiguRE 1. The domain $\Omega_{\epsilon}$

Set $U^{ \pm}=\{x \in U: \pm v(x) \geq 0\}$. Choose a small $c>0$ such that $\{x \in$ $U \cap M: \rho(x) \leq c\} \Subset U$ and each value in $[0, c]$ is a regular value of $\left.\rho\right|_{M \cap U}$. Then $d v$ and $d \rho$ are $\mathbb{R}$-linearly independent at every point of $E=\{x \in M \cap U: \rho(x)=c\}$. Choosing $U$ sufficiently thin around $M \cap U$ we insure that the set $D=\{x \in$ $U: \rho(x) \leq c\}$ has $\mathcal{C}^{2}$ boundary intersecting $M$ transversely along $E$. For small $\epsilon>0$ and $x \in U$ set

$$
\begin{aligned}
v_{\epsilon}^{ \pm}(x) & = \pm v(x)+\epsilon(\rho(x)-c), \\
\Gamma_{\epsilon}^{ \pm} & =\left\{x \in U: v_{\epsilon}^{ \pm}(x)=0\right\}, \\
\Omega_{\epsilon} & =\left\{x \in U: v_{\epsilon}^{+}(x)<0, v_{\epsilon}^{-}(x)<0, \rho(x)<\epsilon\right\} .
\end{aligned}
$$

(See Figure 1,) For sufficiently small $\epsilon>0$ we have $d v_{\epsilon}^{ \pm}= \pm d v+\epsilon d \rho \neq 0$ on $U$; hence $\Gamma_{\epsilon}^{ \pm}$are $\mathcal{C}^{2}$ hypersurfaces satisfying $\Gamma_{\epsilon}^{ \pm} \cap D \subset U^{ \pm}$and $\Gamma_{\epsilon}^{+} \cap \Gamma_{\epsilon}^{-}=E$. As $\epsilon \rightarrow 0$, the domains $\Omega_{\epsilon}$ shrink down to $A$.

We claim that the functions $v_{\epsilon}^{ \pm}$are strongly plurisubharmonic on $\Omega_{\epsilon}$ provided that $\epsilon>0$ is sufficiently small. By the choice of $U$ there is $C_{0}>0$ such that $\left\langle d d^{c} \rho(x), \xi \wedge J \xi\right\rangle \geq C_{0}|\xi|^{2}$ for $x \in U$ and $\xi \in T_{x} X$. Since $j^{1}\left(d d^{c} v\right)$ vanishes on $A$, we have $\left|d d^{c} v(x)\right|=o(|v(x)|+\epsilon)$. (The extra $\epsilon$ takes care of the points near $\Omega_{\epsilon} \cap M \backslash A$.) For $x \in \Omega_{\epsilon}$ we also have

$$
|v(x)| \leq \epsilon|\rho(x)-c| \leq C_{1} \epsilon
$$

with $C_{1}=\sup \{|\rho(x)-c|: x \in D\}$; hence $\left|d d^{c} v(x)\right|=o(\epsilon)$ for $x \in \Omega_{\epsilon}$ as $\epsilon \rightarrow 0$. This gives for $x \in \Omega_{\epsilon}$ and $\xi \in T_{x} X$ :

$$
\begin{aligned}
\left\langle d d^{c} v_{\epsilon}^{ \pm}(x), \xi \wedge J \xi\right\rangle & =\epsilon\left\langle d d^{c} \rho(x), \xi \wedge J \xi\right\rangle \pm\left\langle d d^{c} v(x), \xi \wedge J \xi\right\rangle \\
& \geq\left(C_{0} \epsilon-o(\epsilon)\right) \cdot|\xi|^{2},
\end{aligned}
$$

which is positive for sufficiently small $\epsilon>0$ and for $\xi \neq 0$. This establishes the claim.

Choose a smooth strongly increasing convex function $h:(-\infty, 0) \rightarrow \mathbb{R}$ with $\lim _{t \rightarrow 0} h(t)=+\infty$. The function

$$
\tau(x)=h\left(v_{\epsilon}^{+}(x)\right)+h\left(v_{\epsilon}^{-}(x)\right)+h(\rho(x)-\epsilon), \quad x \in \Omega_{\epsilon}
$$

is then a strongly plurisubharmonic exhaustion function on $\Omega_{\epsilon}$, and hence $\Omega_{\epsilon}$ is Stein according to [38, p. 127, Theorem 5.2.10]. The family $\left\{\Omega_{\epsilon}\right\}$ is therefore a Stein neighborhood basis of $A$ in $X$ satisfying (1.1). 
Remark 3.4. If $v \in \mathcal{C}^{3}(U)$ is a defining function for $M \cap U$ satisfying $\left.j^{1}\left(d d^{c} v\right)\right|_{M \cap U}$ $=0$, then the function $\tau=-\log v+\rho$, defined on $U \cap\{v>0\}$ and tending to $+\infty$ along $M \cap U$, is strongly plurisubharmonic near $A$. Indeed, we have

$$
d d^{c} \tau=-\frac{1}{v} d d^{c} v+\frac{1}{v^{2}} d v \wedge d^{c} v+d d^{c} \rho .
$$

From $\left.j^{1}\left(d d^{c} v\right)\right|_{M}=0$ we infer that the first term vanishes on $M$, the second term is nonnegative since

$$
\left\langle d v \wedge d^{c} v, \xi \wedge J \xi\right\rangle=\langle d v, \xi\rangle\left\langle d^{c} v, J \xi\right\rangle-\langle d v, J \xi\rangle\left\langle d^{c} v, \xi\right\rangle=\langle d v, \xi\rangle^{2}+\langle d v, J \xi\rangle^{2},
$$

and the last term $d d^{c} \rho$ is positive since $\rho$ is strongly plurisubharmonic. The analogous observation holds for $-\log (-v)+\rho$ from the side $\{v<0\}$.

Proof of Theorem 1.1. Choose a small $c>0$ which is a regular value of the function $\left.\rho\right|_{M}$ such that the set $A_{c}=\{x \in M \cap U: \rho(x) \leq c\}$ is compact and the hypothesis concerning the Levi foliation of $M$ still holds on a neighborhood of $A_{c}$. By Proposition 3.1 there exists a $\mathcal{C}^{3}$ defining function $v$ for $M$ near $A_{c}$ satisfying $j^{1}\left(d d^{c} v\right)=0$ on $A_{c}$. Proposition 3.3 now implies that $A_{c}$ is uniformly $\mathcal{H}$-convex and hence a Stein compact. Since there exist arbitrarily small numbers $c>0$ for which the above holds, the set $A=A_{0}$ is a Stein compact as well. This concludes the proof of Theorem 1.1

Theorem 1.4 is obtained in exactly the same way as Theorem 1.1 by combining Proposition 3.1 and the following result.

Proposition 3.5. Let $D$ be a relatively compact, strongly pseudoconvex domain in a Stein manifold $X$, and let $M \subset X$ be a $\mathcal{C}^{3}$-smooth Levi-flat hypersurface intersecting $b D$ transversely. Set $A=M \cap \bar{D}$. If $M$ admits a defining function $v \in \mathcal{C}^{3}(X)$ satisfying $\left.j^{1}\left(d d^{c} v\right)\right|_{A}=0$, then the set $A$, as well as the closure of any connected component of $D \backslash M$, is uniformly $\mathcal{H}$-convex, and hence a Stein compact in $X$.

Proof. Let $D=\{\rho<0\}$ where $\rho$ is a strongly plurisubharmonic function in a neighborhood of $\bar{D}$ in $X$. Assume first that $D \backslash M=D^{+} \cup D^{-}$consists of two connected components $D^{ \pm}$. We may assume that $v>0$ in $D^{+}$locally near $M \cap$ $D$. For sufficiently small $\epsilon>0$ the hypersurface $\Gamma_{\epsilon}^{-}$, constructed in the proof of Proposition 3.3. intersects the hypersurface $\{\rho=\epsilon\}$ transversely. Let $\Omega_{\epsilon}$ denote the domain bounded by $\Gamma_{\epsilon}^{-}$and by the part of $\{\rho=\epsilon\}$ on which $v_{\epsilon}^{-}<0$. (Thus $\Omega_{\epsilon}$ contains $\overline{D^{+}}$but not $\overline{D^{-}}$.) The argument in the proof of Proposition 3.3 shows that $\Gamma_{\epsilon}^{-}$is strongly pseudoconvex and hence $\Omega_{\epsilon}$ is Stein. Thus $\overline{D^{+}}$is uniformly $\mathcal{H}$-convex. The analogous argument applies to any connected component of $M \cap D$, and also to $A$ itself.

\section{Simple foliations}

In this and the following two sections we show how Proposition 1.2 follows from the classical theory of codimension one foliations. We provide sufficient details and references to make the paper accessible to a reader without a substantial background in foliation theory.

By $\mathcal{L}$ we shall always denote a transversely orientable codimension one foliation on a manifold $M$. We begin by recalling some basic facts. 
1. $\mathcal{L}$ is defined by a closed one-form if and only if it admits a foliation atlas whose holonomy cocycle consists of translations in $\mathbb{R}$ ([16, p. 28], [35]).

2. A foliation defined by a closed one-form $\eta$ has trivial leaf holonomy (15, p. 80], 65. Theorem 3.29]). Indeed, if $\gamma$ is a simple closed loop in a leaf $L$, then $\int_{\gamma} \eta=0$ (since $\left.\eta\right|_{L}=0$ ); choose $p \in \gamma$ and define $u(x)=\int_{p}^{x} \eta$ (the integral is independent of the path in a tubular neighborhood of $\gamma$ ); then $d u=\eta \neq 0$ and hence $u$ is a first integral for $\mathcal{L}$ which is injective on a local transversal at $p$, so the germinal holonomy of $L$ along $\gamma$ is trivial. Conversely, a transversely orientable codimension one foliation $\mathcal{L}$ without holonomy and of class $\mathcal{C}^{r}(r \in\{2, \ldots,+\infty\})$ is topologically, but in general not diffeomorphically, conjugate to a $\mathcal{C}^{r}$ foliation defined by a closed nowhere vanishing one-form (Sacksteder [53, [16, $§ 9.2$ and p. 218]).

3. The Godbillon-Vey class of a foliation $\mathcal{L}$ given by a one-form $\eta$ is $g v(\mathcal{L})=$ $[\eta \wedge \alpha \wedge \beta] \in H^{3}(M, \mathbb{R})$ where $\alpha, \beta$ are one-forms satisfying $d \eta=\alpha \wedge \eta, d \alpha=\beta \wedge \eta$ ([16, p. 38], [28, [32], [65, Theorem 2.3]). Clearly $d \eta=0$ implies $g v(\mathcal{L})=0$.

Definition 4.1 ([31, p. 79]). A foliation $\mathcal{L}$ on a manifold $M$ is simple if every point $p \in M$ is contained in a local transversal $\ell \subset M$ to $\mathcal{L}$ which is not intersected more than once by any leaf of $\mathcal{L}$.

We begin the proof of Proposition 1.2 by observing that conditions (a) and (b) are equivalent. Indeed, choosing an open relative neighborhood $U \Subset M$ of $A$ with $\mathcal{C}^{1}$ boundary, the first Betti number of $U$ is finite and hence the condition (b) (that all leaves of $\left.\mathcal{L}\right|_{U}$ are closed) implies that the foliation is simple [31, p. 116, Lemma II.3.9]. The converse is seen by observing that a nonclosed leaf must accumulate on some leaf $L$, and hence it will intersect a local transversal to $L$ infinitely many times.

The implication $(\mathrm{d}) \Rightarrow(\mathrm{a})$ follows from the stability theorems of Thurston and Reeb ([15, p. 78, Theorem 5], [16, §6.2], [31, II.3]). The implication (c) $\Rightarrow$ (a) will be shown in $\S 5$, and (e) is discussed in $\S 6$.

The following result, which was proved for simply connected manifolds by Haefliger and Reeb [36, shows the sufficiency of (a) in Proposition 1.2.

Theorem 4.2. A transversely orientable simple foliation of codimension one and class $\mathcal{C}^{r}(r \in\{2, \ldots,+\infty\})$ is defined in a neighborhood of any compact set by a closed nowhere vanishing one-form of class $\mathcal{C}^{r-1}$.

Globally such a one-form need not exist even if $M=\mathbb{R}^{2}$ [66], and it cannot be chosen to be real analytic even if the foliation is real analytic [30, p. 122].

Having been unable to locate a precise reference for Theorem 4.2, we include a proof following the methods of Haefliger and Reeb [36]. We begin by recalling some results concerning the leaf space of a simple foliation.

Proposition 4.3 (Haefliger and Reeb [36]). Let $\mathcal{L}$ be a simple foliation of codimension one and of class $\mathcal{C}^{r}(r \geq 1)$ on a connected manifold $M$. The space of leaves $Q=M / \mathcal{L}$ carries the structure of a connected, one dimensional, not necessarily Hausdorff $\mathcal{C}^{r}$ manifold such that the quotient projection $\pi: M \rightarrow Q$ is a $\mathcal{C}^{r}$ submersion. The leaf space $Q$ is orientable if and only if $\mathcal{L}$ is transversely orientable, and $Q$ is simply connected if and only if $M$ is such. Every function on $M$ which is constant on the leaves of $\mathcal{L}$ is of the form $f \circ \pi$ for some function $f: Q \rightarrow \mathbb{R}$. 
The $\mathcal{C}^{r}$ structure on the leaf space $Q=M / \mathcal{L}$ is uniquely determined by the requirement that the restriction of the quotient projection $\pi: M \rightarrow Q$ to any local $\mathcal{C}^{r}$ transversal $\ell \subset M$ to the foliation $\mathcal{L}$ is a $\mathcal{C}^{r}$ diffeomorphism of $\ell$ onto $\psi(\ell) \subset Q$.

To prove Theorem 4.2 it suffices to find a nowhere vanishing one-form $\theta$ on the leaf space $Q=M / \mathcal{L}$; its pull-back $\eta=\pi^{*} \theta$ is then a nowhere vanishing one-form on $M$ satisfying ker $\eta=T \mathcal{L}$. A non-Hausdorff manifold $Q$ need not admit any nonconstant $\mathcal{C}^{1}$ functions or one-forms (Wazewski 66] 36]); however, one can do this on compact subsets as we now explain.

A point $q$ in a non-Hausdorff manifold $Q$ is said to be a branch point if there exists another point $q^{\prime} \in Q$ different from $q$ such that $q$ and $q^{\prime}$ have no pair of disjoint neighborhoods; such a pair $\left\{q, q^{\prime}\right\}$ is called a branch pair. (This relation is not transitive.) Branch points of $Q=M / \mathcal{L}$ correspond to separatrices of the foliation $\mathcal{L}$. For a given $q \in Q$ there may exist infinitely many $q^{\prime} \in Q$ such that $\left\{q, q^{\prime}\right\}$ is a branch pair. The main difficulty is that a germ of a smooth function at a branch point need not correspond to the germ of any global function on $Q$ (see an example in [36]). A one dimensional non-Hausdorff manifold $Q$ of class $\mathcal{C}^{r}$ is said to be regular if every germ of a $\mathcal{C}^{r}$ function at any point $q \in Q$ is the germ at $q$ of a global $\mathcal{C}^{r}$ function on $Q$ [36, p. 116, Def. 2]. The following results are due to Haefliger and Reeb.

Proposition 4.4 (36, p. 123, Proposition]). Let $\mathcal{L}$ be a simple $\mathcal{C}^{r}$ foliation of codimension one on a manifold $M$. For any open relatively compact domain $A \subset M$ the leaf space $Q_{A}=A / \mathcal{L}_{A}$ of the restricted foliation $\mathcal{L}_{A}$ is a regular, not necessarily Hausdorff, one dimensional $\mathcal{C}^{r}$ manifold.

Proposition 4.5 ([36, p. 117, Proposition 1]). A regular, simply connected, one dimensional manifold of class $\mathcal{C}^{r}(r \geq 1)$, not necessarily Hausdorff, admits a $\mathcal{C}^{r}$ function with nowhere vanishing differential.

Combining Propositions 4.3, 4.4, and 4.5 one gets the following result which was proved for smooth foliations of $\mathbb{R}^{2}$ by Kamke [39].

Corollary 4.6. Let $r \geq 1$. A simple foliation of codimension one and class $\mathcal{C}^{r}$ on a manifold $M$ admits a noncritical first integral of class $\mathcal{C}^{r}$ on any relatively compact, simply connected open set in $M$.

Assume now that $(M, \mathcal{L})$ satisfies the hypotheses of Theorem 4.2 . Choose a compact set $K$ in $M$. By [31, Lemma IV.1.6] there is a relatively compact domain $A \Subset M$, with $K \subset A$, whose boundary $b A$ is of class $\mathcal{C}^{r}$ and in general position with respect to $\mathcal{L}$, meaning that any local defining function for $\mathcal{L}$ is a Morse function when restricted to $b A$, and distinct critical points of these functions belong to distinct leaves of $\mathcal{L}$ [31, p. 228, Def. IV.1.4].

Note that a point $p \in b A$ is a critical point of a local defining function for $\mathcal{L}$ (restricted to $b A$ ) precisely when the leaf through $p$ meets $b A$ tangentially at $p$ (it is a separatrix). By our choice of $A$ all but finitely many leaves intersect $b A$ transversely, and hence the leaf space $Q_{A}=A / \mathcal{L}_{A}$ has at most finitely many branch points. Furthermore, since distinct points of contact of the leaves with $b A$ belong to distinct leaves, $Q_{A}$ has at most double branch points.

To complete the proof of Theorem 4.2 it suffices to show the following.

Proposition 4.7. An oriented, not necessarily Hausdorff, one dimensional manifold of class $\mathcal{C}^{r}(r \in\{1,2, \ldots,+\infty\})$ which is regular and has at most finitely many 
double branch points (and no multiple branch points) admits a nowhere vanishing differential one-form of class $\mathcal{C}^{r-1}$.

Proof. Fix an orientation on $Q$. Let $x_{j}, y_{j} \in Q(j=1, \ldots, k)$ be all pairs of branch points, i.e., $x_{j}$ and $y_{j}$ cannot be separated by open neighborhoods, but any other pair of distinct points of $Q$ can be separated. For every $j$ we choose an open neighborhood $U_{j} \subset Q$ of $x_{j}$ and an orientation preserving $\mathcal{C}^{r}$ diffeomorphism $h_{j}: U_{j} \rightarrow I=(-1,1) \subset \mathbb{R}$. By regularity of $Q$ we can assume that $h_{j}$ extends to a $\mathcal{C}^{r}$ function $h_{j}: Q \rightarrow \mathbb{R}$. The extended function necessarily has nonzero derivative at the point $y_{j}$ which forms a branch pair with $x_{j}$ [36], and hence $\theta_{j}=d h_{j}$ is a nowhere vanishing one-form of class $\mathcal{C}^{r-1}$ in an open connected neighborhood $V_{j} \subset Q$ of the pair $\left\{x_{j}, y_{j}\right\}$. We may assume that the closures $\bar{V}_{j}$ for $j=1, \ldots, k$ are pairwise disjoint.

Choose a smaller compact neighborhood $E_{j} \Subset V_{j}$ of $\left\{x_{j}, y_{j}\right\}$ such that $V_{j} \backslash E_{j}$ is a union of finitely many segments, none of them relatively compact in $V_{j}$. (We have three segments for a suitable choice of $E_{j}$.) Set $V=\bigcup_{j=1}^{k} V_{j}$ and $E=$ $\bigcup_{j=1}^{k} E_{j}$. Then $Q_{0}=Q \backslash E$ is an open, one dimensional, paracompact, oriented Hausdorff manifold, hence a union of open segments and circles. Any circle in $Q_{0}$ is a connected component of $Q$; choosing a nowhere vanishing one-form on it (in the correct orientation class) does not affect the choices that we shall make on the rest of the set. We do the same on any open segment of $Q_{0}$ which is a connected component of $Q$.

It remains to consider those open segments of $Q_{0}$ which intersect at least one of the sets $V_{j} \backslash E_{j}$. Choose such a segment $J$ and an orientation preserving parametrization $\phi: I=(-1,1) \rightarrow J$. Let $I^{\prime}=\{t \in I: \phi(t) \in V\}$. Then $I^{\prime}$ consists of either one or two subintervals of $I$, each of them having an endpoint at -1 or at +1 . Each of these subintervals is mapped by $\phi$ onto a segment in one of the sets $V_{j} \backslash E_{j}$. (The other possibilities would require a branch point of $Q_{0}$, a contradiction.)

Consider the case when $I^{\prime}=I_{0} \cup I_{1}$ where $I_{0}=(-1, a), I_{1}=(b, 1)$ for a pair of points $-1<a \leq b<1$. Let $j$ and $l$ be chosen such that $\phi\left(I_{0}\right) \subset V_{j}, \phi\left(I_{1}\right) \subset V_{l}$ (we might have $j=l$ ). Note that $\phi(a)$ is an endpoint of $V_{j}$ and $\phi(b)$ is an endpoint of $V_{l}$. Then $\phi^{*} \theta_{j}=d\left(h_{j} \circ \phi\right)$, resp. $\phi^{*} \theta_{l}=d\left(h_{l} \circ \phi\right)$, are nowhere vanishing oneforms on $I_{0}$, resp. on $I_{1}$, both positive with respect to the standard orientation of $\mathbb{R}$. Choose a one-form $\tau$ on $(-1,1)$ which agrees with the above forms near the respective endpoints -1 and +1 (obviously such a $\tau$ exists). Then $\left(\phi^{-1}\right)^{*} \tau$ is a one-form on $J=\phi(I) \subset Q_{0}$ which agrees with $\theta_{j}$ in a neighborhood of $E_{j}$, and it agrees with $\theta_{l}$ in a neighborhood of $E_{l}$. Similarly one deals with the case that $J$ intersects only one of the sets $V_{j}$. Performing this construction for each of the finitely many segments $J \subset Q_{0}$ which intersect $V$ we obtain a nowhere vanishing one-form on $Q$.

Remark 4.8. We wish to elucidate the connection with the methods of Straube and Sucheston 63. Here we constructed a closed one-form by working directly on the leaf space (Proposition 4.7). In 63 the authors look instead for a multiplier $h$ such that $e^{h} \eta$ is a closed one-form defining the Levi foliation $\mathcal{L}$. To understand this, choose a vector field $T$ on $M$ satisfying $\langle\eta, T\rangle=T\rfloor \eta=1$ and set $\alpha=-\operatorname{Lie}_{T}(\eta)$, the Lie derivative of $\eta$ in the direction $-T$. Then $d \eta=\alpha \wedge \eta$, and $\alpha=0$ if $d \eta=0$ [65, Proposition 2.2]. The pull-back $\alpha_{L}$ to a leaf is always closed, and hence it 
defines a de Rham cohomology class $\left[\alpha_{L}\right] \in H^{1}(L ; \mathbb{R})$. From

$$
d\left(e^{h} \eta\right)=e^{h}(d h \wedge \eta+d \eta)=e^{h}(d h+\alpha) \wedge \eta
$$

we infer that $e^{h} \eta$ is closed if and only if $\left.d h\right|_{L}+\alpha_{L}=0$ on every leaf $L$. A solution on $L$ exists if and only if $\left[\alpha_{L}\right]=0 \in H^{1}(L ; \mathbb{R})$, and this holds precisely when $L$ has trivial infinitesimal holonomy. (Indeed, for any closed oriented curve $\gamma \subset L$ we have $\oint_{\gamma} \alpha=\psi_{\gamma}^{\prime}(0)-1$, where $t \rightarrow \psi_{\gamma}(t)$ is the holonomy map of $\gamma$ on a local transversal to $\mathcal{L}$ at a point $p=\psi_{\gamma}(0) \in \gamma$.) Under suitable geometric conditions on $\mathcal{L}$, and without assuming that the foliation extends past $b A$, Straube and Sucheston 63 . found a solution $h$ when $\operatorname{dim} M=3$.

\section{LEVI-FLAT HYPERSURFACES WITHOUT ONE-SIDED HOLONOMY}

The following result shows the sufficiency of condition (c) in Proposition 1.2 ,

Theorem 5.1. Let $M$ be a smooth orientable Levi-flat hypersurface in a complex manifold $X$ such that every element of $\pi_{1}(M)$ is of finite order and its Levi foliation $\mathcal{L}$ has no nontrivial one-sided holonomy (the latter holds in particular if $M$ is real analytic, or if the leaves of $\mathcal{L}$ are simply connected). Then the restriction $\left.\mathcal{L}\right|_{\omega}$ to any open relatively compact subset $\omega \Subset M$ is defined by a closed smooth one-form, and $M$ admits a $\mathcal{C}^{\infty}$ defining function $v$ such that $d d^{c} v$ is flat on $\omega$. If in addition $\omega$ is simply connected, there are an open set $U \subset X$, with $M \cap U=\omega$, and a function $f=u+i v \in \mathcal{C}^{\infty}(U)$ such that $v$ is a defining function for $M \cap U,\left.u\right|_{\omega}$ is a first integral of the Levi foliation $\left.\mathcal{L}\right|_{\omega}$, and the forms $\bar{\partial} f, d d^{c} u$ and $d d^{c} v$ are flat on $\omega$.

The example in $\S 8$ shows that Theorem 5.1 may fail if $M$ is real analytic and $\pi_{1}(M)=\mathbb{Z}$. In general the function $f$ in Theorem 5.1 cannot be chosen to be holomorphic even if $M$ is real analytic and $\bar{\omega}$ is contractible [27.

Proof. Let $\mathcal{L}$ be a smooth, transversely orientable, codimension one foliation of a connected smooth manifold $M$. By Haefliger [35] (see also [31, p. 228, Theorem 1.3]) the existence of a closed, null homotopic transversal $\gamma \subset M$ to $\mathcal{L}$ implies the existence of a leaf $L \in \mathcal{L}$ with nontrivial one-sided holonomy along some closed curve in $L$. (The proof relies on the Poincaré-Bendixson theorem applied to the characteristic foliation induced by $\mathcal{L}$ on a two-disc with boundary $\gamma$.) Hence the conditions in Theorem 5.1 imply that $\mathcal{L}$ does not admit any closed transversal.

Let $\ell \subset M$ be a smooth embedded arc transverse to $\mathcal{L}$. We claim that each leaf $L \in \mathcal{L}$ intersects $\ell$ in at most one point (and hence $\mathcal{L}$ is a simple foliation). If not, we find a subarc $\tau \subset \ell$ whose endpoints $p$ and $q$ belong to the same leaf $L$. Connecting $q$ to $p$ by an arc $\tau^{\prime} \subset L$ we get a closed loop $\lambda=\tau \cdot \tau^{\prime} \subset M$. Using the triviality of the normal bundle to $\mathcal{L}$ along $\tau^{\prime}$ we can modify $\lambda$ in a small tubular neighborhood of $\tau^{\prime}$ into a closed transversal $\widetilde{\lambda}$ to $\mathcal{L}$ [31, p. 228, 1.2. (iii)]. Since we have seen that a closed transversal does not exist under the stated hypotheses, this contradiction proves the claim and shows that $\mathcal{L}$ is a simple foliation.

Theorem 4.2 now implies that $\mathcal{L}$ is defined on any relatively compact open subset $\omega \Subset M$ by a smooth closed one-form $\eta$, and Proposition 3.1 furnishes a smooth defining function $v$ with $d d^{c} v$ flat on $\omega$.

If $\omega$ is simply connected, then $\eta=d u$ for some $u \in \mathcal{C}^{\infty}(\omega)$. Clearly $u$ is constant on the leaves of $\mathcal{L}$, i.e., a first integral of $\left.\mathcal{L}\right|_{\omega}$. Its asymptotic complexification $f=u+i v$ is a smooth function in an open set $U \subset X$ with $U \cap M=\omega$ such that $v$ 
is a defining function for $\omega$ and the forms $\bar{\partial} f, d d^{c} u$ and $d d^{c} v$ are flat on $\omega$ (see the proof of Proposition 3.1).

\section{VANiSHING OF FOLIATION COHOMOLOGY}

Let $(M, \mathcal{L})$ be a $\mathcal{C}^{r}$ foliated manifold. Denote by $\mathcal{C}_{\mathcal{L}}^{r}$ the sheaf of real $\mathcal{C}^{r}$ functions on $M$ which are constant on the leaves. If $\mathcal{L}$ is the Levi foliation of a Levi-flat hypersurface $M$, then $\mathcal{C}_{\mathcal{L}}^{r}$ is the sheaf of real valued CR functions of class $\mathcal{C}^{r}$ on $M$. The following proves Proposition 1.2 in case (e).

Theorem 6.1. Let $\mathcal{L}$ be a transversely orientable codimension one foliation of class $\mathcal{C}^{r}$ on a manifold $M(r \in\{2,3, \cdots, \infty, \omega\})$. If $H^{1}\left(M, \mathcal{C}_{\mathcal{L}}^{r-1}\right)=0$, then $\mathcal{L}$ is given by a closed, nowhere vanishing one-form of class $\mathcal{C}^{r-1}$.

The cohomology group $H^{1}\left(M, \mathcal{C}_{\mathcal{L}}^{r-1}\right)$ can be understood either as a Cech group, or as a de Rham group, [48].

Proof. Transverse orientability of $\mathcal{L}$ implies the existence of a $\mathcal{C}^{r-1}$ vector field $\nu$ which is transverse to $\mathcal{L}$. Choose a transversely oriented $\mathcal{C}^{r}$ foliation atlas $\left\{\left(U_{j}, \phi_{j}\right): j \in J\right\}$ on $M$ defining $\mathcal{L}$. Write $\phi_{j}=\left(\phi_{j}^{\prime}, h_{j}\right)$ where $h_{j}$ maps $U_{j}$ onto an open interval $I_{j} \subset \mathbb{R}$ and $\left\{h_{j}=c\right\}$ are the plaques of $\left.\mathcal{L}\right|_{U_{j}}$. For any $i, j \in J$ with $U_{i j}:=U_{i} \cap U_{j} \neq \emptyset$ we have $h_{i}=\alpha_{i j} \circ h_{j}$ on $U_{i j}$ where $\alpha_{i j}: h_{j}\left(U_{i j}\right) \rightarrow h_{i}\left(U_{i j}\right)$ is a $\mathcal{C}^{r}$ diffeomorphism with positive derivative. (The collection $\left\{\alpha_{i j}\right\}$ is a Haefliger cocycle defining $\mathcal{L}$ [16, 35.) We may assume $\nu\left(h_{j}\right)>0$ for every $j$. Differentiation gives

$$
\nu\left(h_{i}\right)=\left(\alpha_{i j}^{\prime} \circ h_{j}\right) \nu\left(h_{j}\right) \quad \text { on } U_{i j} .
$$

This shows that the collection of positive functions $b_{i j}=\alpha_{i j}^{\prime} \circ h_{j} \in \mathcal{C}^{r-1}\left(U_{i j}\right)$ is a one-cocycle on the covering $\left\{U_{j}\right\}$ with values in the multiplicative sheaf $\mathcal{B}_{\mathcal{L}}^{r-1}$ of positive functions of class $\mathcal{C}^{r-1}$ which are constant on the leaves of $\mathcal{L}$. The exponential map, exp: $\mathcal{C}_{\mathcal{L}}^{r-1} \rightarrow \mathcal{B}_{\mathcal{L}}^{r-1}, b \rightarrow e^{b}$, defines an isomorphism between the two sheaves (the group operation is additive on the first sheaf and multiplicative on the second). The hypothesis $H^{1}\left(M, \mathcal{C}_{\mathcal{L}}^{r-1}\right)=0$ therefore implies that, after passing to a finer $\mathcal{L}$-atlas, the cocycle $b_{i j}$ is a coboundary, $b_{i j}=b_{j} / b_{i}$ for some $b_{j} \in \Gamma\left(U_{j}, \mathcal{B}_{\mathcal{L}}^{r-1}\right)$. This gives

$$
b_{i} \nu\left(h_{i}\right)=b_{j} \nu\left(h_{j}\right) \quad \text { on } U_{i j} .
$$

Since $b_{j}$ is constant on the plaques $\left\{h_{j}=c\right\} \subset U_{j}$, we have $b_{j}=\beta_{j} \circ h_{j}$ for a unique $\mathcal{C}^{r-1}$ function $\beta_{j}: h_{j}\left(U_{j}\right) \rightarrow \mathbb{R}$. Setting $\alpha_{j}=\int \beta_{j}$ and $u_{j}=\alpha_{j} \circ h_{j}$ we have

$$
\nu\left(u_{j}\right)=\left(\alpha_{j}^{\prime} \circ h_{j}\right) \nu\left(h_{j}\right)=\left(\beta_{j} \circ h_{j}\right) \nu\left(h_{j}\right)=b_{j} \nu\left(h_{j}\right)>0 .
$$

We have thus obtained functions $u_{j} \in \mathcal{C}_{\mathbb{R}}^{r}\left(U_{j}\right)(j \in J)$ which are constant on the plaques in $U_{j}$ and satisfy $\nu\left(u_{j}\right)>0$ on $U_{j}$ and $\nu\left(u_{i}\right)=\nu\left(u_{j}\right)$ on $U_{i j}$. Assuming as we may that the sets $U_{i j}$ are connected, it follows that the differences $c_{i j}=u_{j}-u_{i}$ on $U_{i j}$ are real constants. Hence the collection of differentials $d u_{j}(j \in J)$ defines a closed nowhere vanishing one-form $\eta$ on $M$ with $\operatorname{ker} \eta=T \mathcal{L}$.

\section{TRANSVERSE holomorPhiC VeCTOR FIELDS}

In this section we prove Theorems 1.7 1.8 and 1.9. The key ingredient is the following result on the existence of transverse holomorphic vector fields. (Compare with Bedford and Fornæss [11, Proposition 6.2] and Straube and Sucheston [62, p. 156, Proposition].) 
Proposition 7.1. Let $M \subset \mathbb{C}^{n}$ be a Levi-flat hypersurface of class $\mathcal{C}^{r}, r \geq n+2$, with $a \mathcal{C}^{r}$ defining function $v$ satisfying $\left.j^{r-2} d d^{c} v\right|_{M}=0$. Let $A$ be a compact strongly pseudoconvex set in $M$ (see Theorem 1.1). There exists a sequence of holomorphic vector fields $\xi_{\nu}$ in open neighborhoods of $A$ in $\mathbb{C}^{n}(\nu=1,2, \ldots)$ such that the sequence $\xi_{\nu} v=\left\langle d v, \xi_{\nu}\right\rangle$ converges to 1 uniformly on $A$ as $\nu \rightarrow \infty$.

In particular, the holomorphic vector fields $\xi_{\nu}$ are transverse to $M$ near $A$. Recall that a function $v$ with the required properties exists if the Levi foliation of $M$ is defined in a neighborhood of $A$ by a nowhere vanishing closed one-form of class $\mathcal{C}^{r-1}$; see Proposition 1.2 for sufficient conditions.

Proof. By enlarging $A$ slightly inside $M$ we may assume that Proposition 3.3 applies and hence $A$ is uniformly $\mathcal{H}$-convex. Choose Stein open sets $U_{\nu} \supset A$ in $\mathbb{C}^{n}(\nu=$ $1,2,3, \ldots$ ) satisfying (1.1). Let $g_{j}=\partial v / \partial z_{j}$ for $j=1, \ldots, n$. Note that $\partial g_{j} / \partial \bar{z}_{k}=$ $\partial^{2} v / \partial z_{j} \partial \bar{z}_{k}$ is flat to order $r-2$ on $M$; in particular, $g_{j}$ is a CR function on $M$ near $A$.

Lemma 7.2. There exist holomorphic functions $g_{\nu, j} \in \mathcal{O}\left(U_{\nu}\right)(j=1, \ldots, n, \nu=$ $1,2, \ldots)$ satisfying

$$
\left|g_{\nu, j}(z)-g_{j}(z)\right| \leq \text { const } \cdot \nu^{-1 / 2}, \quad z \in A .
$$

Proof. Fix $j \in\{1, \ldots, n\}$ and drop $j$ from the notation. By the assumption we have $|\bar{\partial} g(z)|=o\left(d(z, A)^{r-2}\right)$ where $d(z, A)=\inf \{|z-w|: w \in A\}$. Since every point of $U_{\nu}$ has distance at most $c \nu^{-1}$ from $A$ and the volume of $U_{\nu}$ is proportional to $\nu^{-1}$ (its thickness in the normal direction), we get

$$
\|\bar{\partial} g\|_{L^{\infty}\left(U_{\nu}\right)}=o\left(\nu^{2-r}\right), \quad\|\bar{\partial} g\|_{L^{2}\left(U_{\nu}\right)} \leq\|\bar{\partial} g\|_{L^{\infty}\left(U_{\nu}\right)} \sqrt{\operatorname{Vol}\left(U_{\nu}\right)}=o\left(\nu^{3 / 2-r}\right) .
$$

By Hörmander [38] there exists a solution $u_{\nu}$ of

$$
\bar{\partial} u_{\nu}=\bar{\partial} g \text { on } U_{\nu}, \quad\left\|u_{\nu}\right\|_{L^{2}\left(U_{\nu}\right)} \leq \text { const } \cdot\|\bar{\partial} g\|_{L^{2}\left(U_{\nu}\right)}=o\left(\nu^{3 / 2-r}\right) .
$$

Recall that every $\mathcal{C}^{1}$ function $u$ on $B_{\epsilon}=\left\{\zeta \in \mathbb{C}^{n}:|\zeta|<\epsilon\right\}$ satisfies

$$
|u(0)| \leq \text { const } \cdot\left(\epsilon^{-n}\|u\|_{L^{2}\left(B_{\epsilon}\right)}+\epsilon\|\bar{\partial} u\|_{L^{\infty}\left(B_{\epsilon}\right)}\right) .
$$

Applying this estimate to $u_{\nu}$ at points $z \in A$ with $\epsilon=c \nu^{-1}$ gives

$$
\left|u_{\nu}(z)\right| \leq \nu^{n} o\left(\nu^{3 / 2-r}\right)+\nu^{-1} o\left(\nu^{2-r}\right)=o\left(\nu^{n+3 / 2-r}\right) .
$$

The function $g_{\nu}=g-u_{\nu}$ is then holomorphic on $U_{\nu}$ for every $\nu \in \mathbb{N}$, and assuming $r \geq n+2$ we get the estimate in Lemma 7.2 ,

Now the proof proceeds as in [63, p. 257]. Let $\mathcal{C}(A)$ denote the uniform algebra of all continuous complex valued functions on $A$ endowed with the sup norm, and let $\mathcal{A}(A)$ be the closure in $\mathcal{C}(A)$ of the subalgebra $\left.\mathcal{O}(A)\right|_{A}$ consisting of the restrictions to $A$ of functions holomorphic in open neighborhoods of $A$ in $\mathbb{C}^{n}$. Lemma 7.2 shows that $g_{j} \in \mathcal{A}(A)$ for $j=1, \ldots, n$. Since these functions have no common zeros on $A$, a theorem of Rossi [52, Theorem 2.12] gives functions $f_{j} \in \mathcal{A}(A)$ satisfying

$$
1=\sum_{j=1}^{n} f_{j} g_{j}=\sum_{j=1}^{n} f_{j} \frac{\partial v}{\partial z_{j}}
$$

on $A$. Choose sequences of holomorphic functions $f_{\nu, j}$ in small open neighborhoods of $A$ in $\mathbb{C}^{n}$ such that $\left.\lim _{\nu \rightarrow \infty} f_{\nu, j}\right|_{A}=f_{j}$ for $j=1, \ldots, n$ (the convergence is 
uniform on $A$ ). The sequence of holomorphic vector fields $\xi_{\nu}=\sum_{j=1}^{n} f_{\nu, j} \frac{\partial}{\partial z_{j}}$ then satisfies the conclusion of Proposition 7.1 .

Proof of Theorem 1.7. Let $\Omega \Subset \mathbb{C}^{n}$ be a smoothly bounded pseudoconvex domain with a smooth defining function $\rho$, and let $A \subset b \Omega$ denote the set of infinite type points. Boas and Straube proved in [13, p. 227, Theorem] that the Neumann operator $N_{q}$ and the Bergman projection $P_{q}$ are continuous on the $L_{2}$-Sobolev space $W_{(0, q)}^{s}(\Omega)$ for $0 \leq q \leq n$ and $s \geq 0$ provided that there is a constant $C>1$, and for each sufficiently small $\epsilon>0$ a smooth vector field $\xi_{\epsilon}$ of type $(1,0)$ in an open set $U_{\epsilon} \supset A$ in $\mathbb{C}^{n}$, satisfying the following estimates at each point of $A$ :

(1) $\left|\arg \left(\xi_{\epsilon} \rho\right)\right|<\epsilon, C^{-1}<\left|\xi_{\epsilon} \rho\right|<C$, and

(2) $\left|\left\langle\partial \rho,\left[\xi_{\epsilon}, \partial / \partial \bar{z}_{j}\right]\right\rangle\right|<\epsilon$ for $j=1, \ldots, n$.

(See also 60.) Assume now that the conditions of Theorem 1.7 are satisfied. Let $v$ be an asymptotically pluriharmonic defining function for the Levi-flat hypersurface $M$ in a neighborhood of $A \subset M \cap b \Omega$. Proposition 7.1 furnishes holomorphic vector fields $\xi_{\epsilon}$ satisfying condition (1) for the function $v\left(\right.$ instead of $\rho$ ), and $\left[\xi_{\epsilon}, \partial / \partial \bar{z}_{j}\right]=0$ since $\xi_{\epsilon}$ is holomorphic, so (2) holds. Since $A$ is the closure of its relative interior in $b \Omega$, the gradients of $v$ and $\rho$ are parallel along $A$, and we infer that (1) also holds for $\rho$. The conclusion now follows from the results of 12 and 13 .

If $A$ is not the closure of its relative interior, then $d v$ and $d \rho$ might be completely different on $A$ and we could not infer the condition (1) for $\rho$. This happens in the worm domain where $A$ is a single annular leaf.

Note that a desired family of vector fields satisfying (1) and (2) is obtained in [62 and [63] under weaker hypotheses on $b \Omega$ near the set of infinite type points. In the case at hand the construction in Proposition 7.1 gives a more precise result, although it is based on the same ideas.

Proof of Theorem 1.8. Choose a small open Stein neighborhood $U \subset \mathbb{C}^{n}$ of $A$ and write $U \backslash M=U_{+} \cup U_{-}$, where $U_{ \pm}$are the two sides of $M$ in $U$. We denote by $[M \cap U]^{0,1}$ the $(0,1)$-part of the integration current over $M \cap U$. Let $f$ be a $\mathrm{CR}$ function of class $\mathcal{C}^{k, \alpha}$ in a neighborhood of $\bar{U} \cap M$ in $M$. Then $f[M \cap U]^{0,1}$ is a $\bar{\partial}$-closed current in $U$; since $U$ is Stein, there exists a distribution $F$ on $U$ satisfying $\bar{\partial} F=f[M \cap U]^{0,1}$. As $f[M \cap U]^{0,1}$ is supported by $M \cap U,\left.F\right|_{U_{ \pm}}$is holomorphic on $U_{ \pm}$. Since $f$ is of class $\mathcal{C}^{k, \alpha}$ with $0<\alpha<1$, Theorem 1 in 18 implies that each of the functions $\left.F\right|_{U_{ \pm}}$extends to a function $F_{ \pm} \in \mathcal{C}^{k, \alpha}\left(U_{ \pm} \cup(M \cap U)\right)$, and the jump formula $f=F_{+}-F_{-}$holds on $M \cap U$.

Let $\xi$ be a transverse holomorphic vector field to $M$ in a neighborhood of $A$, furnished by Proposition 7.1. Choosing a correct orientation we may assume that the flow $\phi_{t}$ of $\xi$ carries a neighborhood of $A$ in $M$ to $U_{+}$for small $t>0$, and to $U_{-}$ for small $t<0$. For sufficienty small $t>0$ the function

$$
g_{t}=F_{+} \circ \phi_{t}-F_{-} \circ \phi_{-t}
$$

is well defined and holomorphic in an open neighborhood $V_{t} \subset \mathbb{C}^{n}$ of $A$, and $\left.\lim _{t \downarrow 0} g_{t}\right|_{A}=f$ in the $\mathcal{C}^{k, \alpha}$ topology, thus completing the proof.

If the initial CR function $f$ is of class $\mathcal{C}^{k}$ for some integer $k \geq 1$, the above proof gives an approximation by holomorphic functions in the $\mathcal{C}^{k-0}$ sense. 
Proof of Theorem 1.9. By Proposition 7.1 there is a holomorphic vector field in a neighborhood of $A$ in $\mathbb{C}^{n}$ which is transverse to $M$. A Stein neighborhood basis of $\bar{\Omega}$ is then furnished by [11, Lemma 7.3] (see also [25, Lemma 1]), and the Mergelyan approximation property (2) follows from the work of Fornæss and Nagel [25, Theorem 1].

\section{A LeVi-Flat Hypersurface With A WORM ON EACH SIDE}

The example in this section was suggested to us by J.-E. Fornæss; it was used in a related context by Bedford and Fornæss [11, and the main idea can already be seen in the worm domain of Diederich and Fornæss 23. We shall see that all our results fail in this example due to the existence of an annular leaf with nontrivial holonomy, thus justifying the hypotheses in Theorem 1.1 and Proposition 1.2. (This example was also discussed in [62, p. 147, Remark 4].)

Denote by $(z, w)$ the coordinates on $\mathbb{C}^{*} \times \mathbb{C}$. Let $M \subset \mathbb{C}^{*} \times \mathbb{C}$ be the real analytic hypersurface defined by the following equivalent equations:

$$
M: \quad \Im\left(w e^{i \log z}\right)=0 \Longleftrightarrow \Im\left(w e^{i \log |z|}\right)=0 .
$$

Indeed, the functions inside the parentheses differ only by the positive multiplicative factor $e^{\arg z}$. The first function is multivalued pluriharmonic, and hence $M$ is Leviflat. Introducing the holomorphic map $\Phi: \mathbb{C}^{2} \rightarrow \mathbb{C}^{*} \times \mathbb{C}, \Phi(\zeta, t)=\left(e^{\zeta}, t e^{-i \zeta}\right)$, one sees that $M=\Phi(\mathbb{C} \times \mathbb{R})$. The restriction $\Phi(\cdot, t)$ to a leaf $\mathbb{C} \times\{t\} \subset \mathbb{C} \times \mathbb{R}$ gives a parametrization of the corresponding leaf

$$
L_{t}=\left\{\left(e^{\zeta}, t e^{-i \zeta}\right): \zeta \in \mathbb{C}\right\} \subset M, \quad t \in \mathbb{R}
$$

in the Levi foliation $\mathcal{L}$ of $M$. This parametrization is biholomorphic if $t \neq 0$ (so $L_{t} \simeq \mathbb{C}$ ) while for $t=0$ it is the covering map $\mathbb{C} \rightarrow L_{0}=\mathbb{C}^{*} \times\{0\}, \zeta \rightarrow\left(e^{\zeta}, 0\right)$.

The line $E=\{(1, s): s \in \mathbb{R}\} \subset M$ is a global transversal for $\mathcal{L}$, and for every $t \in \mathbb{R}$ we have $L_{t} \cap E=\left\{\left(1, t e^{2 k \pi}\right): k \in \mathbb{Z}\right\}$. The only closed leaf is $L_{0}=\mathbb{C}^{*} \times\{0\}$ to which all other leaves $L_{t}$ approach spirally. Identifying $E$ with $\mathbb{R}$ we see that the holonomy of $L_{0}$ along the positively oriented circle $|z|=1$ is $s \rightarrow s e^{-2 \pi}$. The space of leaves $M / \mathcal{L}$ is the union of a point representing $L_{0}$ and two closed circles, each representing the leaves $L_{t}$ for $t>0$, resp. for $t<0$. Writing $h(z, w)=w e^{i \log z}$ we see that the closed holomorphic one-form

$$
\eta=\frac{d h}{h}=i \frac{d z}{z}+\frac{d w}{w}
$$

on $\mathbb{C}^{*} \times \mathbb{C}^{*}$ defines the Levi foliation of $M \backslash L_{0}$; there is no such closed one-form in any neighborhood of $L_{0}$ due to nontrivial holonomy.

The hypersurface $M$ divides $\mathbb{C}^{*} \times \mathbb{C}$ into two connected components

$$
M_{ \pm}=\left\{(z, w) \in \mathbb{C}^{*} \times \mathbb{C}: \pm \Im\left(w e^{i \log |z|}\right)>0\right\}
$$

which have the essential properties of a worm domain (Diederich and Fornæss [23]). The family of complex annuli

$$
R_{s}=\left\{(z, i s): e^{-\pi / 2}<|z|<e^{\pi / 2}\right\}, \quad s \in \mathbb{R}
$$

satisfies the following:

$-R_{0} \subset L_{0} \subset M$,

- $b R_{s} \subset M$ for all $s \in \mathbb{R}$,

$-R_{s} \subset M_{+}$if $s>0$ and $R_{s} \subset M_{-}$if $s<0$. 
If $f$ is a holomorphic function in an open neighborhood of the annular set

$$
A_{0}=\left\{(z, w) \in M: e^{-\pi / 2} \leq|z| \leq e^{\pi / 2},|w| \leq 1\right\},
$$

then by analytic continuation along the family of annuli $R_{s}, s \in[-1,1]$, one obtains a holomorphic extension of $f$ to a neighborhood of the Levi-flat hypersurface $R=\bigcup_{s \in[-1,1]} R_{s}$, which therefore belongs to the holomorphic hull of $A_{0}$. Since $R$ intersects both $M_{+}$and $M_{-}$, we see that Theorem 1.1 fails. Likewise Theorem 1.4 fails for any strongly pseudoconvex domain $D \subset \mathbb{C}^{*} \times \mathbb{C}$ containing $A_{0}$, and Theorem 1.9 fails for any pseudoconvex domain $\Omega \Subset \mathbb{C}^{*} \times \mathbb{C}$ with $A_{0} \subset b \Omega$.

Corollary 1.6 fails as well, which is seen as follows. With $D$ as above set $D_{ \pm}=$ $D \cap M_{ \pm}, A=\bar{D} \cap M \supset A_{0}, \omega=b D_{+} \backslash A, K=\bar{D}_{-}$and $\Omega=D \backslash \widehat{K} \subset D_{+}$, where $\widehat{K}$ denotes the $\mathcal{O}\left(\bar{D}_{+}\right)$-hull of $K$. The above discussion shows that $R \subset \widehat{K}$ and hence $\Omega$ is a proper subset of $D_{+}$. By [41] and [46] every continuous CR function on $\omega$ extends holomorphically to $\Omega$; since $\Omega$ is pseudoconvex [59], there exists $f \in \mathcal{O}(\Omega) \cap \mathcal{C}(\Omega \cup \omega)$ which does not extend holomorphically to $D_{+}$.

Finally, if $\Omega \Subset \mathbb{C}^{2}$ is a smooth pseudoconvex domain with $A_{0} \subset b \Omega$ and such that $b \Omega \backslash A_{0}$ is strongly pseudoconvex, then the results of Barrett 8 carry over from the standard worm domain and show that the $\bar{\partial}$-Neumann problem is not globally regular on $\Omega$ [62, p. 147, Remark 4]. For a more precise result see Christ [19].

In the above example the worm phenomenon is caused by an annulus contained in a leaf with nontrivial infinitesimal holonomy; there exist small holomorphic deformations of this annulus with boundaries contained in $M$ and such that the interiors move to both sides of $M$. We now show that this cannot happen along a leaf with trivial (first order) infinitesimal holonomy. Recall that the holonomy along a loop $\gamma \subset L$ is infinitesimally trivial to order $k \in \mathbb{N}$ if the associated holonomy map on a local transversal $\ell$ to $\mathcal{L}$ at some point $p \in \gamma$ is of the form $x \rightarrow x+o\left(|x|^{k}\right)$ in some (and hence any) local coordinate $x$ on $\ell$, with $x=0$ corresponding to $p$.

Proposition 8.1. Let $M$ be an orientable Levi-flat hypersurface in a complex manifold $X$. Assume that $K \subset X$ is a Stein compact which is contained in a leaf $L$ of the Levi foliation of $M$. If the holonomy of $L$ is infinitesimally trivial to the first order along each loop contained in an open neighborhood of $K$ in $L$, then there is a holomorphic vector field in a neighborhood of $K$ in $X$ which is transverse to $M$.

Translating $M$ by the flow of a vector field in Proposition 8.1 gives a Stein neighborhood basis of a compact set $A \subset M$ which contains $K$ in its relative interior; in particular, $M$ has no worm in a neighborhood of $K$.

Proof. Replacing $X$ by a small Stein neighborhood of $K$ we may assume that the leaf $L$ is a closed complex submanifold of $X$. Let $\mathcal{J}$ denote the sheaf of ideals of $L$. For a fixed $k \in \mathbb{N}$ let $\mathcal{P}$ be the subsheaf of $\mathcal{J}$ consisting of all germs $h_{p} \in \mathcal{J}_{p}$ $(p \in L)$ such that $d h(p) \neq 0$ and $\left.\Im h\right|_{M}=o\left(|h|^{k}\right)$ on a neighborhood of $p$ in $M$; for $p \notin L$ we take $\mathcal{P}_{p}=\mathcal{J}_{p}=\mathcal{O}_{p}$. Let $\widetilde{\mathcal{P}}$ denote the image of $\mathcal{P}$ in $\mathcal{J} / \mathcal{J}^{k+1}$. If the holonomy of $L$ is trivial to order $k$, then the sheaf $\widetilde{\mathcal{P}}$ admits a section $\tilde{h}$ (Barrett 6 , p. 361, Proposition]). In our case this holds with $k=1$. Since $X$ is Stein, Cartan's Theorem B implies that $\tilde{h}$ lifts to a section $h$ of $\mathcal{J}$, i.e., $h$ is a holomorphic function vanishing on $L$ to the first order and satisfying $\Im h=o(|h|)$ on a neighborhood of the leaf $L$ in $M$. Choose a holomorphic vector field $\xi$ on $X$ satisfying $\langle d h, \xi\rangle=\sqrt{-1}$ on $L$. From $\left.\Im h\right|_{M}=o(|h|)$ we see that $\xi$ is transverse to $M$ along $L$. 


\section{Piecewise Real analytic Levi-Flat hypersurfaces}

In $\S 8$ we have seen that the conclusion of Theorem 1.1 may fail in the presence of a leaf with nontrivial infinitesimal holonomy, but Proposition 8.1 gives some hope that leaves with trivial infinitesimal holonomy may present no problem. Hence the following is a reasonable problem.

Problem 9.1. Suppose that the Levi foliation of $M$ is simple in the complement of finitely many leaves $L_{1}, \ldots, L_{k}$ and the infinitesimal holonomy of each $L_{j}$ is trivial (perhaps to a high order). Does it follow that each compact set $A \subset M$ as in Theorem 1.1 is a Stein compact?

In this section we give examples indicating that foliations of this type are rather common in the class of piecewise real analytic Levi-flat hypersurfaces. We also show that some of the standard constructions of real codimension one foliations, such as the creation of a Reeb component, turbulization and spinning, can be performed in this class.

Let $\Sigma$ be a real analytic manifold. Suppose that $\Sigma=D_{1} \cup \cdots \cup D_{m}$ where each $D_{j}$ is a closed domain with real analytic boundary $b D_{j}$ in $\Sigma$ such that for $j \neq k$ the intersection $D_{j} \cap D_{k}$ is a union of connected components of $b D_{j}$ and $b D_{k}$ (possibly empty). Assume that for each $j$ we are given a real analytic codimension one foliation $\mathcal{F}_{j}$ in an open neighborhood of $D_{j}$ in $\Sigma$ such that $b D_{j}$ is a union of leaves. Let $\mathcal{F}$ denote the foliation of $\Sigma$ whose restriction to $D_{j}$ equals $\left.\mathcal{F}_{j}\right|_{D_{j}}$. If the foliations on each pair of adjacent domains $D_{j}, D_{k}$ match up to order $r$ along their common boundary components, then $\mathcal{F}$ is a transversely piecewise real analytic foliation of class $\mathcal{C}^{r}$ of $\Sigma$, with real analytic leaves. (This is a special case of the tangential gluing of foliations [16, §3.4].) Recall that a pair of codimension one foliations match up to order $r$ along a common boundary leaf $L$ if and only if their holonomies along any closed loop $\gamma \subset L$ are tangent to the identity map to order $r$ on the respective sides of a local transversal to $L$ at a point in $\gamma$ [16, p. 91, Proposition 3.4.2]. $\mathcal{F}$ is transversely orientable when each of the constituent foliations is such and their orientations match along the boundary leaves.

Suppose now that $\Sigma$ is embedded in a complex manifold $(X, J)$ as a real analytic maximal real submanifold, meaning that $T_{x} X=T_{x} \Sigma \oplus J\left(T_{x} \Sigma\right)$ for all $x \in \Sigma$. (Every real analytic manifold $\Sigma$ admits such an embedding into its complexification. If $\operatorname{dim}_{\mathbb{R}} \Sigma=3$ and $\Sigma$ is orientable, then it admits a maximal real embedding in $\mathbb{C}^{3}[4$, [26], 33.) Let $\mathcal{F}$ be a real analytic codimension one foliation of $\Sigma$. Complexifying a leaf $F \in \mathcal{F}$ gives a complex submanifold $\widetilde{F}$ in an open neighborhood of $\Sigma$, with $\widetilde{F} \cap \Sigma=F$ and $\operatorname{dim}_{\mathbb{C}} \widetilde{F}=\operatorname{dim}_{\mathbb{R}} F$. Locally we can perform the complexification uniformly for all nearby leaves; restricting our attention to a compact subset $A \subset \Sigma$ we thus find an open neighborhood $U \subset X$ of $A$ and a Levi-flat real analytic hypersurface $M \subset U$ with Levi foliation $\mathcal{L}=\{\widetilde{F} \cap U: F \in \mathcal{F}\}$. Along $\Sigma \cap U \subset M$ we have $T M=T \mathcal{F} \oplus J(T \mathcal{F}) \oplus N=T \mathcal{L} \oplus N$ where $N \simeq T \Sigma / T \mathcal{F}$ is the normal bundle of $\mathcal{F}$ in $\Sigma$.

Essentially the same construction applies to a piecewise real analytic foliation $\mathcal{F}$ of $\Sigma$ described above. If $\mathcal{F}$ is transversely $\mathcal{C}^{r}$ at each of the leaves in $b D_{j} \cap b D_{k}$, then the complexifications of the individual foliations $\mathcal{F}_{j}$ on $D_{j}$ match to order $r$ along the common boundary components. In this way we obtain a Levi foliation $\mathcal{L}$ on a piecewise real analytic $\mathcal{C}^{r}$ hypersurface $M=M_{1} \cup \cdots \cup M_{m}$, with $\left.\mathcal{L}\right|_{M_{j}}$ the complexification of $\mathcal{F}_{j}$. The foliated manifolds $(M, \mathcal{L})$ and $(\Sigma, \mathcal{F})$ have the 
same structure, in particular, the same space of leaves. We now turn to concrete examples.

9.1. A Levi-flat hypersurface with a Reeb foliation. (For the standard $\mathcal{C}^{\infty}$ Reeb foliation, see Examples 1.1.12 and 3.3.11 in [16.) Choose integers $n, r \geq 1$. Let $(x, t)$ be coordinates on $\mathbb{R}^{n} \times S^{1}$. Choose a strictly increasing polynomial function $\lambda: \mathbb{R} \rightarrow \mathbb{R}$ satisfying $\lambda(0)=-\pi / 2, \lambda(1)=0, \lambda(4)=\pi / 2$, whose derivatives up to order $r$ vanish at $0,1,4$. The one-form

$$
\omega=\cos \lambda(\rho) d \rho+\sin \lambda(\rho) d t \quad\left(\rho=|x|^{2} \in \mathbb{R}_{+}\right)
$$

is real analytic and nowhere vanishing on $\mathbb{R}^{n} \times S^{1}$. From $d \omega \wedge \omega=0$ we infer that $\omega$ determines a codimension one foliation $\mathcal{F}$ of $\mathbb{R}^{n} \times S^{1}$. Its restriction to $T=D^{n} \times S^{1}$ $\left(D^{n}=\left\{x \in \mathbb{R}^{n}:|x| \leq 1\right\}\right)$ is a Reeb foliation of $T$ (a Reeb component) with the only closed leaf $F_{0}=b D^{n} \times S^{1}=S^{n-1} \times S^{1}$ to which all other leaves spirally approach. This foliation is not defined by a closed one-form in any neighborhood of $F_{0}$ due to nontrivial holonomy along the loops $\{x\} \times S^{1}(|x|=1)$; this holonomy is flat to order $r$. The restriction of $\mathcal{F}$ to $\{|x|<1\} \times S^{1}$ is a simple foliation given by a fibration over $S^{1}$.

We decompose the three-sphere $S=S^{3}$ in a union $T_{1} \cup T_{2}$ of two solid tori diffeomorphic to $D^{2} \times S^{1}$, with $T_{1} \cap T_{2}=b T_{1}=b T_{2}=F_{0} \simeq S^{1} \times S^{1}$. Endowing each $T_{j}$ with a Reeb foliation $\mathcal{F}_{j}$ described above, one obtains a piecewise real analytic Reeb foliation $\mathcal{F}$ of $S$ whose Reeb components match to order $r \in \mathbb{N}$ along the boundary leaf $F_{0}$. (For the $\mathcal{C}^{\infty}$ case see [16, p. 93, Example 3.4.4.].) Embedding $S$ as a real analytic totally real submanifold of $\mathbb{C}^{3}[1$ and complexifying $\mathcal{F}$, we obtain a piecewise real analytic Levi-flat hypersurface $M \subset \mathbb{C}^{3}$ whose Levi foliation has the structure of the Reeb foliation on $S^{3}$. $M$ admits an asymptotically defining function in the complement of the leaf $L_{0}=\widetilde{F}_{0}$ (the complexification of the torus leaf $F_{0} \subset S$ ), but there is no such function near $L_{0}$ due to nontrivial holonomy.

By Novikov [50] every $\mathcal{C}^{2}$ foliation of $S^{3}$ by surfaces contains a Reeb component. According to Barrett [6] the Reeb foliation on $S^{3}$ cannot be realized as the Levi foliation of a smooth compact Levi-flat hypersurface, but there are topological realizations with a corner along the torus leaf.

9.2. Turbulization. Let $\mathcal{F}$ be the foliation in the previous example, but considered now on $\{|x| \leq 2\} \times S^{1}$. Since $\lambda(4)=\pi / 2$ and the derivatives of $\lambda$ up to order $r$ vanish at 4 , the one-form $\omega$ is tangent to $d t$ to order $r$ along the torus $T^{\prime}=\{|x|=2\} \times S^{1}$, and hence $\mathcal{F}$ matches along $T^{\prime}$ to order $r$ with the trivial (horizontal) foliation $\mathcal{F}_{0}$ of $\mathbb{R}^{n} \times S^{1}$ with leaves $\{t=c\}$. Let $\mathcal{F}_{\text {turb }}$ denote the foliation of $\mathbb{R}^{n} \times S^{1}$ which equals $\mathcal{F}$ on $\{|x| \leq 2\} \times S^{1}$ and equals $\mathcal{F}_{0}$ on $\{|x| \geq 2\} \times S^{1}$. This deformation of $\mathcal{F}_{0}$, known as turbulization [16, can be made in a small tubular neighborhood of any closed transversal $\gamma$ in a codimension one foliation and produces a new Reeb component along $\gamma$. In the real analytic case it can be made by a piecewise real analytic deformation which is suitable for complexification, thus giving Levi-flat realizations of turbulization.

9.3. Spinning. The spinning modification can be made at a boundary component $S \subset b M$ of a foliated manifold $(M, \mathcal{F})$ provided that every leaf of $\mathcal{F}$ intersects $S$ transversely and the induced foliation $\{F \cap S: F \in \mathcal{F}\}$ is determined by a closed one-form on $S$ [16, p. 84, Example 3.3.B]. This modification changes $S$ into a closed leaf of a new foliation $\mathcal{F}_{\text {spin }}$ which is then suitable for tangential gluing along $S$. 
If all data are real analytic, then $\mathcal{F}_{\text {spin }}$ can be made piecewise real analytic and smooth to a given finite order. In fact, the Reeb component $\mathcal{F}$ on $D^{n} \times S^{1}$ in $\S 9.1$ is obtained by spinning the trivial foliation with leaves $D^{n} \times\{t\}$ along the boundary $b D^{n} \times S^{1}$.

\section{ACKNOWLEDGEMENTS}

We thank J.-E. Fornæss for telling us about the example in $\S 8$, E. Straube for pointing out the connection with the $\bar{\partial}$-Neumann problem and for very helpful discussions, G. Tomassini for suggesting Theorem 1.8 and J. Mrčun, T. Ohsawa and M.-C. Shaw for helpful remarks. We are indebted to the referee for several suggestions which helped us to improve the exposition.

\section{REFERENCES}

1. AHERN, P., RUDIN, W., Hulls of 3-spheres in $\mathbb{C}^{3}$, in The Madison Symposium on Complex Analysis (Madison, WI, 1991, 1-27), Contemp. Math., 137, Amer. Math. Soc., Providence, RI, 1992. MR1190966 (93k:32020)

2. AIRAPETIAN, R. A., Extending CR functions from piecewise smooth CR manifolds. Math. Sb., 134 (1987), 108-118 (Russian).

3. ANDREOTTI, A., NACINOVICH, M., Analytic Convexity. Ann. Sc. Norm. Sup. Pisa, 7 (1980), 287-372. MR581145 (81m:32025)

4. AUDIN, M., Fibrés normaux d'immersions en dimension double, points doubles d'immersions lagrangiennes et plongements totalement réels. Comment. Math. Helv., 63 (1988), 593-623. MR $966952(89 \mathrm{~m}: 57032)$

5. BAOUENDI, M. S., TRÈVES, F., A property of the functions and distributions annihilated by a locally integrable system of complex vector fields. Ann. of Math., (2) 113 (1981), 387-421. MR607899 (82f:35057)

6. BARRETT, D. E., Complex analytic realization of Reeb's foliation of $S^{3}$. Math. Z., 203 (1990), 355-361. MR1038705 (91f:32018)

7. Global convexity properties of some families of three-dimensional compact Levi-flat hypersurfaces. Trans. Amer. Math. Soc., 332 (1992), 459-474. MR1055805 (93c:32026)

8. Behavior of the Bergman projection on the Diederich-Fornæss worm. Acta Math., 168 (1992), 1-10. MR.1149863 (93c:32033)

9. BARRETT, D. E., FORNÆSS, J.-E., On the smoothness of Levi-foliations. Publ. Mat., 32 (1988), 171-177. MR975896 (90b:32037)

10. BEDFORD, E., DE BARTOLOMEIS, P., Levi flat hypersurfaces which are not holomorphically flat. Proc. Amer. Math. Soc., 81 (1981), 575-578. MR601733(82a:32025)

11. BEDFORD, E., FORNÆSS, J. E., Domains with pseudoconvex neighborhood systems. Invent. Math., 47 (1978), 1-27. MR0499316 (58:17215)

12. BOAS, H. P., STRAUBE, E., Sobolev estimates for the $\bar{\partial}$-Neumann operator on domains in $\mathbb{C}^{n}$ admitting a defining function that is plurisubharmonic at the boundary. Math. Z., 206 (1991), 81-88. MR1086815 (92b:32027)

13. estimates for the $\bar{\partial}$-Neumann problem. J. Geom. Anal., 3 (1993), 225-235. MR1086815 (92b:32027)

14. BOGGESS, A., CR Manifolds and the Tangential Cauchy-Riemann Complex. CRC Press, Boca Raton, 1991. MR1211412 (94e:32035)

15. CAMACHO, C., LINS NETO, A., Geometric Theory of Foliations. Birkhäuser, Boston, 1985. MR:824240(87a:57029)

16. CANDEL, A., CONLON, L., Foliations I. Grad. Studies in Math., 23, Amer. Math. Soc., Providence, Rhode Island, 2000. MR1732868 (2002f:57058)

17. CHEN, S.-C., SHAW, M.-C., Partial Differential Equations in Several Complex Variables. Amer. Math. Soc. and International Press, Providence, RI, 2001. MR.1800297 (2001m:32071)

18. CHIRKA, E. M., Analytic representation of CR functions. (Russian) Math. USSR Sbornik, 27 (1975), 526-553. 
19. CHRIST, M., Global $\mathbb{C}^{\infty}$ irregularity of the $\bar{\partial}$-Neumann problem for worm domains. J. Amer. Math. Soc., 9 (1996), 1171-1185. MR.1370592 (96m:32014)

20. COLŢOIU, M., Complete locally pluripolar sets. J. Reine Angew. Math., 412 (1990), 108-112. MR:1074376 (91h:32010)

21. D'ANGELO, J. P., Real hypersurfaces, orders of contact, and applications. Annals of Math., 115 (1982), 615-637. MR657241 (84a:32027)

22. DEMAILLY, J.-P., Cohomology of $q$-convex spaces in top degrees. Math. Z., 204 (1990), 283-295. MR1055992 (91e:32014)

23. DIEDERICH, K., FORNÆSS, J.-E., Pseudoconvex domains: An example with nontrivial Nebenhülle. Math. Ann., 225 (1977), 275-292. MR0430315 (55:3320)

24. FOlland, G. B., KOHN, J. J., The Neumann Problem for the Cauchy-Riemann Complex. Annals of Math. Studies 75, Princeton Univ. Press, 1972. MR0461588 (57:1573)

25. FORNÆSS, J. E., NAGEL, A., The Mergelyan property for weakly pseudoconvex domains. Manuscripta Math., 22 (1977), 199-208. MR0457779 (56:15983)

26. FORSTNERIČ, F., On totally real embeddings into $\mathbb{C}^{n}$. Expo. Math., 4 (1986), 243-255. MR.880125 (88g:32016)

27. A contractible Levi-flat hypersurface in $\mathbb{C}^{2}$ which is a determining set for pluriharmonic functions. Arkiv Math. 44 (2006), 87-91. MR2237212 (2007c:32047)

28. GHYS, É., L'invariant de Godbillon-Vey. Astérisque, 177-178 (1989), 155-181. MR 1040572 (91h:57015)

29. GIGANTE, G., TOMASSINI, G., Foliations with complex leaves. Diff. Geom. Appl., 5 (1995), 33-49. MR 1319934 (96b:32024)

30. GODBILlON, C., Dynamical Systems on Surfaces. Springer-Verlag, Berlin, 1983. MR681119 (84b:57018)

31. Feuilletages, études géométriques. Birkhäuser, Basel-Boston-Berlin, 1991. MR:1120547 (93i:57038)

32. GODBILLON, C., VEY, J., Un invariant des feuilletages de codimension 1. C. R. Acad. Sci. Paris, Sér. A, 273 (1971), 92-95. MR0283816 (44:1046)

33. GROMOV, M., Partial Differential Relations. Ergebnisse der Mathematik und ihrer Grenzgebiete (3), 9. Springer, Berlin-New York, 1986. MR864505 (90a:58201)

34. GUNNING, R. C., ROSSI, H., Analytic Functions of Several Complex Variables. PrenticeHall, Englewood Cliffs, NJ, 1965. MR0180696 (31:4927)

35. HAEFLIGER, A., Structures feuilletées et cohomologie à valeur dans un faisceau de groupoïdes. Comment. Math. Helv., 32 (1958), 248-329. MR0100269 (20:6702)

36. HAEFLIGER, A., REEB, G., Variétés (non séparées) à un dimension et structures feuilletées du plan. L'Enseignement Math., 3 (1957), 107-125. MR0089412 (19:671c)

37. HENKIN, G. M., LEITERER, J., Theory of functions on complex manifolds. AkademieVerlag, Berlin, 1984. MR774049 (86a:32002)

38. HÖRMANDER, L. An Introduction to Complex Analysis in Several Variables, 3rd ed. North Holland, Amsterdam, 1990. MR1045639 (91a:32001)

39. KAMKE, E., Über die partielle Differentialgleichung $f z_{x}+g z_{y}=h$. Math. Z., 41 (1936), 56-66; 42 (1936), 287-300. MR 1545604

40. KOHN, J. J., A survey of the $\bar{\partial}-$ Neumann problem. Proc. Symp. Pure Math., 41, pp. 137-145. Amer. Math. Soc., Providence, R.I., 1984. MR740877 (85e:32023)

41. LAURENT-THIÉBAUT, C., Sur l'extension des fonctions CR dans une variété de Stein. Ann. Mat. Pura Appl., 150 (1988), 141-151. MR946033(89j:32020)

42. _ Extension de formes différentielles CR. C. R. Acad. Sci. Paris Sér. I Math., 306 (1988), 539-542. MR0941620 (89e:32039)

43. __ Sur l'équation de Cauchy-Riemann tangentielle dans une calotte strictement pseudoconvexe. Internat. J. Math., 16 (2005), 1063-1079. MR2180065 (2006m:32044)

44. LAURENT-THIÉBAUT, C., LEITERER, J., On the Hartogs-Bochner extension phenomenon for differential forms. Math. Ann., 284 (1989), 103-119. MR995385 (90c:32026)

45. LAURENT-THIÉBAUT, C., PORTEN, E., Analytic extension from non-pseudoconvex boundaries and $A(D)$-convexity. Ann. Inst. Fourier (Grenoble), 53 (2003), 847-857. MR2008443 (2004h:32041)

46. LUPACCIOLU, G., Characterization of removable sets in strongly pseudoconvex boundaries. Arkiv Math., 32 (1994), 455-473. MR1318542 (96a:32027) 
47. LUPACCiOlU, G., TOMASSini, G., An extension theorem for CR-functions. Ann. Mat. Pura Appl., 137 (1984), 257-263. MR772261 (86e:32021)

48. MASA, X., Alexander-Spanier cohomology of foliated manifolds. Illinois J. Math., 46 (2002), 979-998. MR1988246 (2004c:57043)

49. NEMIROVSKI, S., Stein domains with Levi-flat boundaries on compact complex surfaces. Mat. Zametki, 66 (1999), 632-634. English transl.: Math. Notes, 66 (1999), 522-525. MR.1747093 (2001d:32025)

50. NOVIKOV, S. P., Topology of foliations. Trans. Moscow Math. Soc., 14 (1965), 268-305. MR0200938 (34:824)

51. OHSAWA, T., On the Levi-flats in complex tori of dimension two. Publ. Res. Inst. Math. Sci. 42 (2006), 361-377. MR2250063

52. ROSSI, H., Holomorphically convex sets in several complex variables. Annals Math., 24 (1961), 470-493. MR0133479 (24:A3310)

53. SACKSTEDER, R., Foliations and pseudogroups. Amer. J. Math., 87 (1965), 79-102. MR0174061 (30:4268)

54. SHAW, M.-C., $L^{p}$ estimates for local solutions of $\bar{\partial}_{\mathrm{b}}$ on strongly pseudo-convex CR manifolds. Math. Ann., 288 (1990), 35-62. MR1070923 (92b:32028)

55 . L L L L L existence theorems with estimates for $\bar{\partial}_{b}$ on weakly pseudo-convex CR manifolds. Math. Ann., 294 (1992), 677-700. MR1190451 (94b:32026)

56. SHCHERBINA, N. V., Decomposition of a common boundary of two domains of holomorphy into analytic curves. Iszvestia Akad. Nauk SSSR Ser. Mat, 46 (1982), 1106-1123. English transl.: Math. USSR Iszvestia, 21 (1983), 399-413. MR675533 (84b:32018)

57. SIU, Y.-T., Every Stein subvariety admits a Stein neighborhood. Invent. Math., 38 (1976), 89-100. MR0435447 (55:8407)

58. —, $\bar{\partial}$-regularity for weakly pseudoconvex domains in compact Hermitian symmetric spaces with respect to invariant metrics. Ann. Math., (2) 156 (2002), 595-621. MR 1933078 (2003h:32057)

59. SLODKOWSKI, Z., Analytic set-valued functions and spectra. Math. Ann., 256 (1981), 363386. MR626955 (83b:46070)

60. STRAUBE, E. J., A sufficient condition for global regularity of the $\bar{\partial}$-Neumann operator. Preprint, 2005. [arXiv:math.CV/0510354]

61. A A A L International Congress of Mathematicians, Madrid, 2006.

62. STRAUBE, E. J., SUCHESTON, M. K., Plurisubharmonic defining functions, good vector fields, and exactness of a certain one-form. Monatsh. Math., 136 (2002), 249-258. MR1919648 (2003f:32051)

63. Levi foliations in pseudoconvex boundaries and vector fields that commute approximately with $\bar{\partial}$. Trans. Amer. Math. Soc., 355 (2002), 143-154. MR1928081(2003h:32058)

64. SULLIVAN, D., Cycles for the dynamical study of foliated manifolds and complex manifolds. Invent. Math., 36 (1976), 225-255. MR0433464 (55:6440)

65. TONDEUR, P., Geometry of Foliations. Birkhäuser, Boston, 1997. MR1456994 (98d:53037)

66. WAZEWSKY, T., Sur l'équation $P p+Q q=0$. Mathematica, 8 (1934), 103-116; 9 (1935), 179-182.

Institute of Mathematics, Physics and Mechanics, University of Ljubljana, JadranSKa 19, 1000 Ljubljana, Slovenia

E-mail address: franc.forstneric@fmf.uni-lj.si

Institut Fourier, UMR 5582 CNRS/UJF, BP 74, 38402 St. Martin d’Hères Cedex, France

E-mail address: Christine.Laurent@ujf-grenoble.fr 